Research Article

\title{
Exact Values of Zagreb Indices for Generalized T-Sum Networks with Lexicographic Product
}

\author{
Jia-Bao Liu $\mathbb{D}^{1},{ }^{1}$ Sana Akram, ${ }^{2}$ Muhammad Javaid ${ }^{\circ},{ }^{2}$ and Zhi-Ba Peng ${ }^{1}$ \\ ${ }^{1}$ School of Mathematics and Physics, Anhui Jianzhu University, Hefei 230601, China \\ ${ }^{2}$ Department of Mathematics, School of Science, University of Management and Technology, Lahore, Pakistan
}

Correspondence should be addressed to Muhammad Javaid; javaidmath@gmail.com

Received 16 April 2021; Accepted 17 August 2021; Published 31 August 2021

Academic Editor: Huseyin Isik

Copyright (c) 2021 Jia-Bao Liu et al. This is an open access article distributed under the Creative Commons Attribution License, which permits unrestricted use, distribution, and reproduction in any medium, provided the original work is properly cited.

The use of numerical numbers to represent molecular networks plays a crucial role in the study of physicochemical and structural properties of the chemical compounds. For some integer $k$ and a network $G$, the networks $S_{k}(G)$ and $R_{k}(G)$ are its derived networks called as generalized subdivided and generalized semitotal point networks, where $S_{k}$ and $R_{k}$ are generalized subdivision and generalized semitotal point operations, respectively. Moreover, for two connected networks, $G_{1}$ and $G_{2}, G_{1}\left[G_{2}\right]_{S_{k}}$ and $G_{1}\left[G_{2}\right]_{R_{k}}$ are $T$-sum networks which are obtained by the lexicographic product of $T\left(G_{1}\right)$ and $G_{2}$, respectively, where $T \varepsilon\left\{S_{k}, R_{k}\right\}$. In this paper, for the integral value $k \geq 1$, we find exact values of the first and second Zagreb indices for generalized $T$-sum networks. Furthermore, the obtained findings are general extensions of some known results for only $k=1$. At the end, a comparison among the different generalized $T$-sum networks with respect to first and second Zagreb indices is also included.

\section{Introduction}

Topological index (TI) being a molecular descriptor is a mathematical measure that associates a molecular network with a real number and predicts the underlying molecular network's biological, chemical, and structural properties. Molecular descriptors were used by Wiener [1] and Trinjastic and Gutman and Trinajstić [2] to determine the boiling point of paraffin and the total $\pi$-electron energy of the molecules, respectively. TIs are also used in the study of cheminformatics to classify molecules in terms of quantitative structure behavior and property relationships. Most notably, all TIs are invariants under the networks' isomorphism parameter [3, 4]. Many TIs exist in the literature for networks. Degree-based TIs, distance-based TIs, and polynomial-based TIs are the three major types of these. The degrees-based TIs are more familiar than the others [5].

In the field of chemical network theory, networks operations are frequently used to discover the new families of networks. Yan et al. [6] explained the subdivision $\left(S_{1}\right)$ and semitotal point $\left(R_{1}\right)$ operations on a molecular network $G$ and attain the Wiener indices of the consequent networks
$S_{1}(G)$ and $R_{1}(G)$. After that, Eliasi and Taeri [7] explained the $T$-sum network $G_{1}+{ }_{T} G_{2}$ by using Cartesian product of $G_{1}$ and $S\left(G_{2}\right)$, where $T \varepsilon\left\{S_{1}, R_{1}\right\}$. Deng et al. [8] defined the $T$-sum network $\left(G_{1}\left[G_{2}\right]\right)$ with the help of lexicographic product and also calculated the first and second Zagreb indices, and Akhter and Imran [9] also calculated the forgotten index of the $T$-sum networks. For more studies of the TIs under the operations of networks, see $[10,11]$.

Recently, for some integer $k \geq 1$, Liu et al. [12] defined the generalized subdivision $\left(S_{k}\right)$ and generalized semitotal point $\left(R_{k}\right)$ operations. They also computed the 1 st and 2 nd Zagreb indices for T-sum networks with the help of Cartesian product, i.e., $\left(G_{1}+_{T_{k}} G_{2}\right)$ for $T_{k} \varepsilon\left\{S_{k}, R_{k}\right\}[13,14]$. In this study, we find the exact values of the Zagreb indices for the generalized $T$-sum networks which are constructed with the help of generalized subdivision, generalized semitotal point operations, and lexicographic product. In the remaining paper, Section 1 has some previous knowledge related to our work and Section 2 has some basic definition. The key findings are presented in Section 3, and the conclusion and applications of these indices are presented in Section 4. 


\section{Preliminaries}

We consider undirected, connected, and simple networks, where $V(G)$ is a vertex set and $E(G) \subseteq V(G) \times V(G)$ is an edge set. In addition, $|V(G)|=n$ is order and $|E(G)|=m$ is size of $\mathrm{G}$. Each vertices of a molecular network is referred to as an atom, and edges reflect the bonding between atoms.

The number of incident edges is called its degree. The networks $V\left(P_{n}\right)=\left\{b_{j}: 1 \leq j \leq n\right\}$ and $E\left(P_{n}\right)=\left\{b_{j} b_{j+1}\right.$ : $1 \leq j \leq n\}, V\left(C_{n}\right)=\left\{b_{j}: 1 \leq j \leq n\right\} \& E\left(C_{n}\right)=\left\{b_{i} b_{i+1}: 1 \leq\right.$ $j \leq n-1\} \cup\left\{b_{n} b_{1}\right\}$ and $V\left(K_{n}\right)=\left\{b_{i}: 1 \leq j \leq n\right\}$, and $E\left(K_{n}\right)=$ $\left\{b_{i} b_{j}: 1 \leq i, j \leq n\right\}$ are called path $\left(P_{n}\right)$, cycle $\left(C_{n}\right)$, and complete $\left(K_{n}\right)$, respectively [3].

Definition 1. Let $G_{1}$ and $G_{2}$ be two networks with their vertex sets as $V\left(G_{1}\right)=\left\{y_{1}, y_{2}, \ldots, y_{n}\right\}$ and $V\left(G_{2}\right)=\left\{z_{1}, z_{2}, \ldots, z_{n}\right\}$, respectively. The Cartesian product $G_{1} \times G_{2}$ of these networks is defined as follows:

$$
\begin{aligned}
& V\left(G_{1} \times G_{2}\right)=V\left(G_{1}\right) \times V\left(G_{2}\right), \\
& E\left(G_{1} \times G_{2}\right)=\left\{\left(y_{1}, z_{1}\right)\left(y_{2}, z_{2}\right):\left(y_{1}, z_{1}\right),\left(y_{2}, z_{2}\right) \in V\left(G_{1}\right) \times\left(G_{2}\right)\right\},
\end{aligned}
$$

with conditions either $y_{1}=y_{2}$ in $V\left(G_{1}\right)$ and $z_{1} z_{2} \in E\left(G_{2}\right)$ or $y_{1} y_{2} \in E\left(G_{1}\right)$ and $z_{1}=z_{2}$ in $V\left(G_{2}\right)$.

$$
\begin{aligned}
& V\left(G_{1}\left[G_{2}\right]\right)=V\left(G_{1}\right) \times V\left(G_{2}\right), \\
& E\left(G_{1}\left[G_{2}\right]\right)=\left\{\left(y_{1}, z_{1}\right)\left(y_{2}, z_{2}\right):\left(y_{1}, z_{1}\right),\left(y_{2}, z_{2}\right) \in V\left(G_{1}\right)\left[\left(G_{2}\right)\right]\right\},
\end{aligned}
$$

Definition 2. Let $G_{1}$ and $G_{2}$ be two networks with their vertex sets as $V\left(G_{1}\right)=\left\{y_{1}, y_{2}, \ldots, y_{n}\right\}$ and $V\left(G_{2}\right)=\left\{z_{1}, z_{2}\right.$, $\left.\ldots, z_{n}\right\}$, respectively. Then, the lexicographic product $G_{1}\left[G_{2}\right]$ of these networks is defined as with conditions either. $y_{1}=y_{2}$ in $V\left(G_{1}\right)$ and $z_{1} z_{2} \in E\left(G_{2}\right)$ or $y_{1} y_{2} \in E\left(G_{1}\right)$ and $z_{1}, z_{2}$ in $V\left(G_{2}\right)$.

For integer $k \geq 1$, Liu et al. [12] defined the following graphs using the generalized subdivision and semitotal point operations:

(i) $S_{k}(G)$ network is found out by inserting $k$-vertices in each edge of $G$

(ii) $R_{k}(G)$ is found out from $S_{k}(G)$ by connecting the old vertices which are adjacent in $G$

Eliasi and Taeri [7] proposed the generalized $T$-sum networks under the operation of two connected networks $G_{1}$

$$
\begin{aligned}
V\left(G_{1}\left[G_{2}\right]\right)_{T_{k}} & =V\left(T_{k}\left(G_{1}\right)\right) \times V\left(G_{2}\right), \\
E\left(G_{1}\left[G_{2}\right]\right)_{T_{k}} & =\left\{\left(y_{1}, z_{1}\right)\left(y_{2}, z_{2}\right):\left(y_{1}, z_{1}\right),\left(y_{2}, z_{2}\right) \in V\left(G_{1}\right)\left[\left(G_{2}\right)\right]_{T_{k}}\right\},
\end{aligned}
$$

with conditions either $y_{1}=y_{2}$ in $V\left(T_{k}\left(G_{1}\right)\right)$ and $z_{1} z_{2} \in E\left(G_{2}\right)$ or $y_{1} y_{2} \in E\left(T_{k}\left(G_{1}\right)\right)$ and $z_{1}, z_{2}$ in $V\left(G_{2}\right)$.

For more explanation, see Figures 1-4 .

\section{Main Results}

In this part, we will learn about the Zagreb indices of T-sum networks obtained by the operations of generalized subdivision and generalized semitotal point and lexicographic product. and $G_{2}$ based on the Cartesian product. Deng et al. [8] defined the generalized $T$-sum networks under the operation of two connected networks $G_{1}$ and $G_{2}$ based on the Lexicographic product as follows.

Definition 3. Let $G_{1}$ and $G_{2}$ be two networks and $T_{k} \varepsilon\left\{S_{k}, R_{k}\right\}$ and $T_{k}\left(G_{1}\right)$ be a network with vertex set $V\left(T_{k}\left(G_{1}\right)\right)$ and edge set $E\left(T_{k}\left(G_{1}\right)\right)$. Then, the generalized $T_{k}$-sum network $G_{1}\left[G_{2}\right]_{T_{k}}$ is a graph with the vertex set:
Theorem 1. Let $G_{1}$ and $G_{2}$ be two networks; then, for some integer $k$, the first Zagreb index of $S_{k}$-sum network is

$$
\begin{aligned}
M_{1}\left(G_{1}\left[G_{2}\right]_{S_{k}}\right)= & 8 n_{2} e_{1} e_{2}+n_{1} M_{1}\left(G_{2}\right)+n_{2}^{3} M_{1}\left(G_{1}\right) \\
& +4 e_{1} n_{2}^{3}+4 e_{1} n_{2}^{2}(k-1)++8 n_{2} e_{1}(k-1) \\
& \sum_{i=1}^{n_{2}-1}\left(n_{2}-i\right) .
\end{aligned}
$$




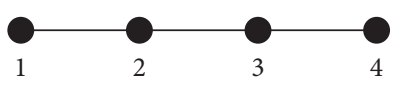

(a)

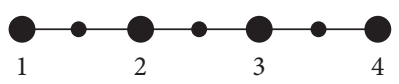

(b)

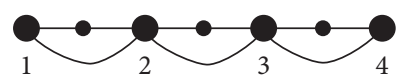

(c)

Figure 1: (a) $G \cong P_{4},(\mathrm{~b}) S_{1}(G) \cong S_{1}\left(P_{4}\right)$, and $(\mathrm{c}) R_{1}(G) \cong R_{1}\left(P_{4}\right)$.

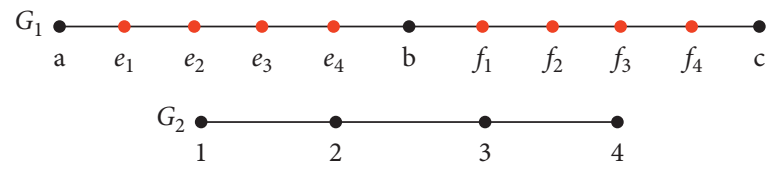

Figure 2: (a) $S\left(G_{1}\right) \cong S\left(P_{3}\right)$ and $(\mathrm{b}) G_{2} \cong P_{4}$.
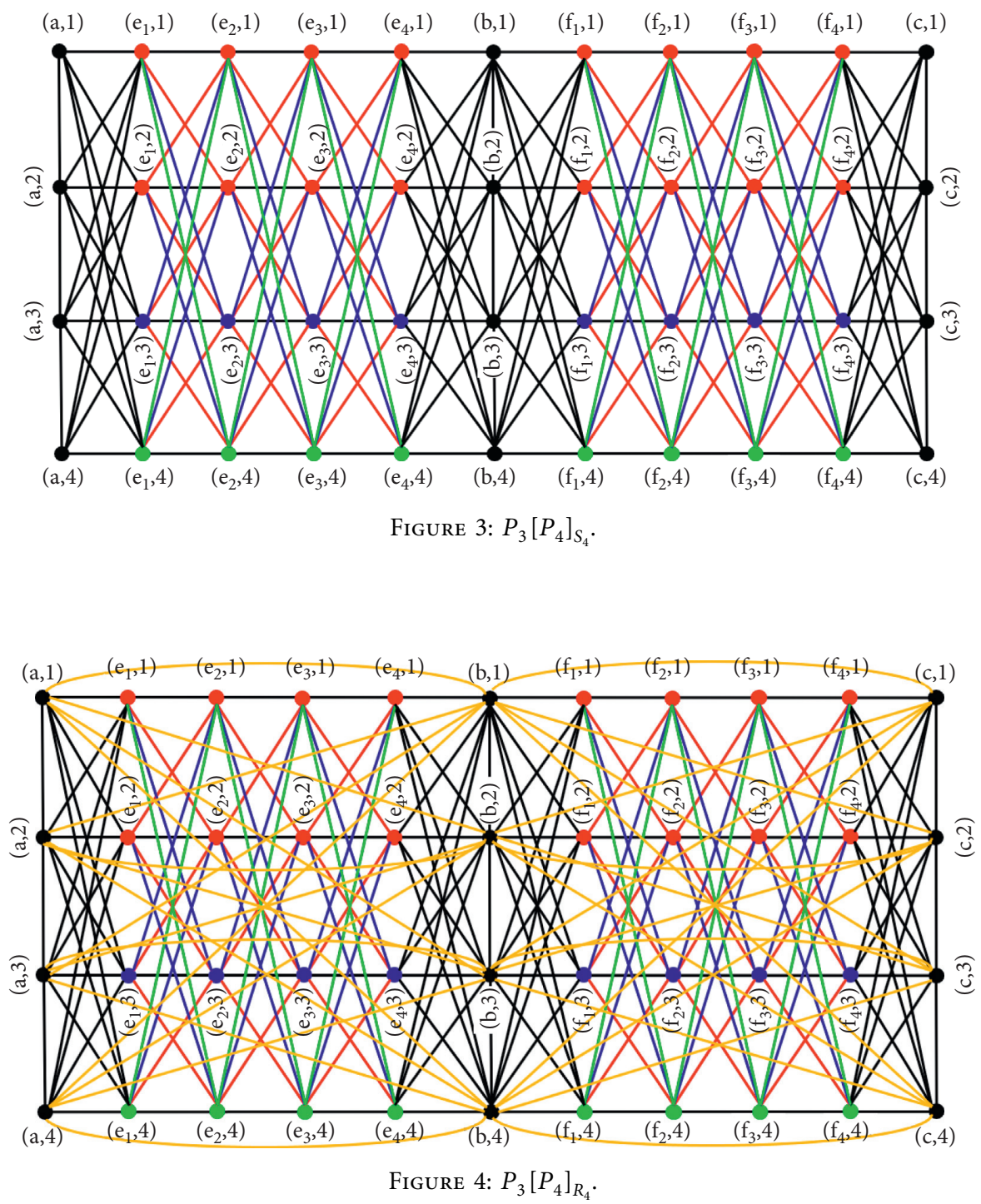
Proof. Let $d(u, v)=d_{G_{1}\left[G_{2}\right]_{S_{S}}}(u, v)$ be the degree of a vertex $(u, v)$ in the network $G_{1}\left[G_{2}\right]_{S_{k}}^{k}$ :

$$
\begin{aligned}
M_{1}\left(G_{1}\left[G_{2}\right]_{S_{k}}\right)= & \sum_{(u, v) \varepsilon V\left(G_{1}\left[G_{2}\right]_{S_{k}}\right)} d^{2}(u, v)=\sum_{\left(u_{1}, v_{1}\right)\left(u_{2}, v_{2}\right) \varepsilon E\left(G_{1}\left[G_{2}\right]_{s_{k}}\right)}\left[d\left(u_{1}, v_{1}\right)+d\left(u_{2}, v_{2}\right)\right] \\
= & \sum_{u_{1}=u_{2}=u \varepsilon V\left(G_{1}\right)} \sum_{v_{1} v_{2} \varepsilon E\left(G_{2}\right)}\left[d\left(u, v_{1}\right)+d\left(u, v_{2}\right)\right]+\sum_{v_{1} \varepsilon V\left(G_{2}\right)} \sum_{v_{2} \varepsilon V\left(G_{2}\right) u_{1} u_{2} \varepsilon E\left(S_{k}\left(G_{2}\right)\right)}\left[d\left(u_{1}, v_{1}\right)+d\left(u_{2}, v_{2}\right)\right] \\
& +\sum_{v_{1}=v_{2}=v \varepsilon V\left(G_{2}\right)} \sum_{u_{1} u_{2} \varepsilon E\left(G_{1}\right)}\left[d\left(u_{1}, v_{1}\right)+d\left(u_{2}, v_{2}\right)\right] \\
& \sum_{u_{1} u_{2} \varepsilon V\left(S_{k}\left(G_{1}\right)\right)-V\left(G_{1}\right)}\left[\sum _ { \substack { v _ { 1 } \varepsilon V ( G _ { 2 } ) \\
= } } \sum _ { v _ { 2 } \varepsilon V ( G _ { 2 } ) u _ { 1 } u _ { 2 } \varepsilon E ( S _ { k } ( G _ { 2 } ) ) } \left[d+\sum 2+\sum 3+\sum 4 .\right.\right.
\end{aligned}
$$

Now, first, we calculate

$$
\begin{aligned}
& \sum 1=\sum_{u_{1}=u_{2}=u \varepsilon V\left(G_{1}\right)} \sum_{v_{1} v_{2} \varepsilon E\left(G_{2}\right)}\left[d\left(u, v_{1}\right)+d\left(u, v_{2}\right)\right] \\
& =\sum_{u \varepsilon V\left(G_{1}\right)} \sum_{v_{1} v_{2} \varepsilon E\left(G_{2}\right)}\left[n_{2} d_{G_{1}}(u)+d_{G_{2}}\left(v_{1}\right)+n_{2} d_{G_{1}}(u)+d_{G_{2}}\left(v_{2}\right)\right] \\
& =\sum_{u \varepsilon V\left(G_{1}\right)} \sum_{v_{1} v_{2} \varepsilon E\left(G_{2}\right)}\left[2 n_{2} d_{G_{1}}(u)+d_{G_{2}}\left(v_{1}\right)+d_{G_{2}}\left(v_{2}\right)\right] \\
& =\sum_{u \varepsilon V\left(G_{1}\right)} e_{2} \cdot 2 n_{2} d(u)+\sum_{u \varepsilon V\left(G_{1}\right)} M_{1}\left(G_{2}\right)=2 e_{1} e_{2}\left(2 n_{2}\right)+n_{1} M_{1}\left(G_{2}\right)=4 n_{2} e_{1} e_{2}+n_{1} M_{1}\left(G_{2}\right), \\
& \sum 2=\sum_{v_{1} \varepsilon V\left(G_{2}\right)} \sum_{v_{2} \varepsilon V\left(G_{2}\right)} \sum_{u_{1} u_{2} \varepsilon E\left(S_{k}\left(G_{1}\right)\right)}\left[d\left(u_{1}, v_{1}\right)+d\left(u_{2}, v_{2}\right)\right] \\
& =\sum_{v_{1} \varepsilon V\left(G_{2}\right)} \sum_{v_{2} \varepsilon V\left(G_{2}\right)} \sum_{u \varepsilon\left(v\left(G_{1}\right)\right), a \varepsilon\left(E\left(G_{1}\right)\right)}\left[d\left(u, v_{1}\right)+d\left(a, v_{2}\right)\right] \\
& u \text { and } a \text { are incident } \\
& =\sum_{v_{1} \varepsilon V\left(G_{2}\right) v_{2} \varepsilon V\left(G_{2}\right)} \sum \quad\left[n_{2} d_{G_{1}} u+d_{G_{2}} v_{1}+2 n_{2}\right] \\
& u \varepsilon\left(v\left(G_{1}\right)\right), a \varepsilon\left(E\left(G_{1}\right)\right) \\
& =\sum_{v_{1} \varepsilon V\left(G_{2}\right)} \sum_{v_{2} \varepsilon V\left(G_{2}\right)} \sum_{u \varepsilon\left(v\left(G_{1}\right)\right)}\left[d_{G_{1}} u\right]\left[n_{2} d_{G_{1}} u+d_{G_{2}} v_{1}+2 n_{2}\right] \\
& =n_{2} n_{2} n_{2} \sum_{u \varepsilon\left(v\left(G_{1}\right)\right)}\left[d_{G_{1}}^{2} u\right]+\sum_{v_{1} \varepsilon V\left(G_{2}\right)} \sum_{v_{2} \varepsilon V\left(G_{2}\right)} 2 e_{1}\left[d_{G_{2}} v_{1}+2 n_{2}\right] \\
& =n_{2}^{3} M_{1}\left(G_{1}\right)+2 e_{1}\left[2 e_{2} n_{2}+2 n_{2} n_{2} n_{2}\right]=n_{2}^{3} M_{1}\left(G_{1}\right)+4 n_{2} e_{1} e_{2}+4 e_{1} n_{2}^{3},
\end{aligned}
$$




$$
\begin{aligned}
\sum 3 & =\sum_{v_{1}=v_{2}=v \varepsilon V} \sum_{\left(G_{2}\right)} \sum_{u_{1} u_{2} \varepsilon E\left(G_{1}\right)}\left[d\left(u_{1}, v_{1}\right)+d\left(u_{2}, v_{2}\right)\right] \\
& =n_{2}(k-1) u_{2} \varepsilon V\left(S_{k}\left(G_{1}\right)\right)-V\left(G_{1}\right) \\
\sum 4 & =\sum_{v_{1} \varepsilon V\left(G_{2}\right)} \sum_{v_{2} \varepsilon V\left(G_{2}\right)} \sum_{u_{1} u_{2} \varepsilon E\left(s_{k}\left(G_{1}\right)\right)}\left[d\left(u_{1}, v_{1}\right)+d\left(u_{2}, v_{2}\right)\right] \\
& =\left[2 n_{2}+2 n_{2}\right] \sum_{i=1}^{n_{2}-1}\left(2 n_{2}-2 i\right)(k-2) e_{1}+\left[2 n_{2}+2 n_{2}\right] \sum_{i=1}^{n_{2}-1}\left(2 n_{2}-2 i\right) e_{1} \\
& =8 n_{2} \sum_{i=1}^{n_{2}-1}\left(n_{2}-i\right)(k-2) e_{1}+8 n_{2} \sum_{i=1}^{n_{2}-1}\left(n_{2}-i\right) e_{1}=8 n_{2} e_{1}(k-1) \sum_{i=1}^{n_{2}-1}\left(n_{2}-i\right) .
\end{aligned}
$$

So, the result is

$$
\begin{aligned}
= & 8 n_{2} e_{1} e_{2}+n_{1} M_{1}\left(G_{2}\right)+n_{2}^{3} M_{1}\left(G_{1}\right)+4 e_{1} n_{2}^{3} \\
& +4 e_{1} n_{2}^{2}(k-1)+8 n_{2} e_{1}(k-1) \sum_{i=1}^{n_{2}-1}\left(n_{2}-i\right) .
\end{aligned}
$$

Theorem 2. Let $G_{1}$ and $G_{2}$ be two networks; then, for some integer $k$, the second Zagreb index of $S_{k}$-sum network is

$$
\begin{aligned}
M_{2}\left[G_{1}\left[G_{2}\right]_{S_{k}}\right]= & n_{2}^{2} e_{2} M_{1}\left(G_{1}\right)+2 n_{2} e_{1} M_{1}\left(G_{2}\right)+n_{1} M_{2}\left(G_{2}\right)+2 n_{2}^{4} M_{1}\left(G_{1}\right)+8 n_{2}^{2} e_{1} e_{2}+4 e_{1} n_{3}^{2}(k-1) \\
& +8 n_{2}^{2} e_{1}(k-1) \sum_{i=1}^{n_{2}-1}\left(n_{2}-i\right) .
\end{aligned}
$$

Proof. Let $d(u, v)=d_{G_{1}\left[G_{2}\right]_{s_{k}}}(u, v)$ be the degree of a vertex $(u, v)$ in the graph $G_{1}\left[G_{2}\right]_{S_{k}}$ :

$$
\begin{aligned}
M_{1}\left(G_{1}\left[G_{2}\right]_{S_{k}}\right) & =\sum_{(u, v) \varepsilon V\left(G_{1}\left[G_{2}\right]_{S_{k}}\right)} d^{2}(u, v)=\sum_{\left(u_{1}, v_{1}\right)\left(u_{2}, v_{2}\right) \varepsilon E\left(G_{1}\left[G_{2}\right]_{S_{k}}\right)}\left[d\left(u_{1}, v_{1}\right) d\left(u_{2}, v_{2}\right)\right] \\
& =\sum_{u_{1}=u_{2}=u \varepsilon V} \sum_{\left(G_{1}\right)}\left[d\left(u, v_{1}\right) d\left(u, v_{2}\right)\right]+\sum_{v_{1} \varepsilon E\left(G_{2}\right)} \sum_{v_{2} \varepsilon V\left(G_{2}\right)} \sum_{u_{1} u_{2} \varepsilon E\left(S\left(G_{1}\right)\right)}\left[d\left(u_{1}, v_{1}\right) d\left(u_{2}, v_{2}\right)\right] \\
& =\sum_{u_{1}=u_{2}=u \varepsilon V\left(G_{1}\right)} \sum_{v_{1} v_{2} \varepsilon E\left(G_{2}\right)}\left[d\left(u, v_{1}\right)+d\left(u, v_{2}\right)\right]+\sum_{v_{1} \varepsilon V\left(G_{2}\right)} \sum_{v_{2} \varepsilon V\left(G_{2}\right)} \sum_{u_{1} u_{2} \varepsilon E\left(S_{k}\left(G_{2}\right)\right)}\left[d\left(u_{1}, v_{1}\right)+d\left(u_{2}, v_{2}\right)\right] \\
& =\sum 1+\sum 2+\sum 3+\sum 4 .
\end{aligned}
$$


Now, first, we calculate

$$
\begin{aligned}
& \sum 1=\sum_{u_{1}=u_{2}=u \varepsilon V\left(G_{1}\right)} \sum_{v_{1} v_{2} \varepsilon E\left(G_{2}\right)}\left[d\left(u, v_{1}\right) d\left(u, v_{2}\right)\right] \\
& =\sum_{u \varepsilon V\left(G_{1}\right)} \sum_{v_{1} v_{2} \varepsilon E\left(G_{2}\right)}\left[n_{2}^{2} d_{G_{1}}^{2}(u)+n_{2} d_{G_{1}}(u)\left[d_{G_{2}}\left(v_{1}\right)+d_{G_{2}}\left(v_{2}\right)\right]+d_{G_{2}}\left(v_{1}\right) d_{G_{2}}\left(v_{2}\right)\right] \\
& =\sum_{u \varepsilon V\left(G_{1}\right)}\left[n_{2}^{2} e_{2} d_{G_{1}}^{2}(u)+n_{2} d_{G_{1}}(u) M_{1}\left(G_{2}\right)+M_{2}\left(G_{2}\right)\right]=n_{2}^{2} e_{2} M_{1}\left(G_{1}\right)+2 n_{2} e_{1} M_{1}\left(G_{2}\right)+n_{1} M_{2}\left(G_{2}\right), \\
& \sum 2=\sum_{v_{1} \varepsilon V\left(G_{2}\right)} \sum_{v_{2} \varepsilon V\left(G_{2}\right)} \sum_{u_{1} u_{2} \varepsilon E\left(S_{k}\left(G_{1}\right)\right)}\left[d\left(u_{1}, v_{1}\right) d\left(u_{2}, v_{2}\right)\right] \\
& =\sum_{v_{1} \varepsilon V\left(G_{2}\right)} \sum_{v_{2} \varepsilon V\left(G_{2}\right)} \sum_{\substack{u \varepsilon\left(v\left(G_{1}\right)\right), a \varepsilon\left(E\left(G_{1}\right)\right) \\
u \text { and } a \text { are incident }}}\left[d\left(u, v_{1}\right) d\left(a, v_{2}\right)\right] \\
& =\sum_{v_{1} \varepsilon V\left(G_{2}\right)} \sum_{v_{2} \varepsilon V\left(G_{2}\right)} \sum_{\substack{u \varepsilon\left(v\left(G_{1}\right)\right), a \varepsilon\left(E\left(G_{1}\right)\right) \\
u \text { and } a \text { are incident }}}\left[n_{2} d_{G_{1}}(u)+d_{G_{2}}\left(v_{1}\right)\right]\left[2 n_{2}\right] \\
& =n_{2} n_{2} 2 n_{2} \sum_{u \varepsilon\left(v\left(G_{1}\right)\right)}\left[d_{G_{1}}^{2}(u)\right]+\sum_{v_{1} \varepsilon V\left(G_{2}\right)} \sum_{v_{2} \varepsilon V\left(G_{2}\right)} 2 e_{1}\left[2 n_{2} d_{G_{2}}\left(v_{1}\right)\right] \\
& =2 n_{2}^{4} M_{1}\left(G_{1}\right)+2 n_{2} n_{2} 2 e_{1} 2 e_{2}=2 n_{2}^{4} M_{1}\left(G_{1}\right)+8 n_{2}^{2} e_{1} e_{2}, \\
& \sum 3=\sum_{v_{1}=v_{2}=v \mathcal{E} V\left(G_{2}\right)} \sum_{u_{1} u_{2} \varepsilon E\left(G_{1}\right)}\left[d\left(u_{1}, v_{1}\right) d\left(u_{2}, v_{2}\right)\right] \\
& u_{1} u_{2} \varepsilon V\left(S_{k}\left(G_{1}\right)\right)-V\left(G_{1}\right) \\
& =n_{2}(k-1) e_{1}\left[2 n_{2} 2 n_{2}\right]=4 e_{1} n_{3}^{2}(k-1), \\
& \sum 4=\sum_{v_{1} \varepsilon V\left(G_{2}\right)} \sum_{v_{2} \varepsilon V\left(G_{2}\right)} \sum_{u_{1} u_{2} \varepsilon E\left(S_{k}\left(G_{1}\right)\right)}\left[d\left(u_{1}, v_{1}\right) d\left(u_{2}, v_{2}\right)\right] \\
& =\left[2 n_{2} \times 2 n_{2}\right] \sum_{i=1}^{n_{2}-1}\left(2 n_{2}-2 i\right)(k-2) e_{1}+\left[2 n_{2} \times 2 n_{2}\right] \sum_{i=1}^{n_{2}-1}\left(2 n_{2}-2 i\right) e_{1} \\
& =8 n_{2}^{2} \sum_{i=1}^{n_{2}-1}\left(n_{2}-i\right)(k-2) e_{1}+8 n_{2}^{2} \sum_{i=1}^{n_{2}-1}\left(n_{2}-i\right) e_{1}=8 n_{2}^{2} e_{1}(k-1) \sum_{i=1}^{n_{2}-1}\left(n_{2}-i\right) \text {. }
\end{aligned}
$$

So, the final result is

$$
=n_{2}^{2} e_{2} M_{1}\left(G_{1}\right)+2 n_{2} e_{1} M_{1}\left(G_{2}\right)+n_{1} M_{2}\left(G_{2}\right)+2 n_{2}^{4} M_{1}\left(G_{1}\right)+8 n_{2}^{2} e_{1} e_{2}+4 e_{1} n_{3}^{2}(k-1)+8 n_{2}^{2} e_{1}(k-1) \sum_{i=1}^{n_{2}-1}\left(n_{2}-i\right) \text {. }
$$

Theorem 3. Let $G_{1}$ and $G_{2}$ be two networks; then, for some integer $k$, the first Zagreb index of $R_{k}$-sum network is

$$
M_{1}\left(G_{1}\left[G_{2}\right]_{R_{k}}\right)=16 n_{2} e_{1} e_{2}+n_{1} M_{1}\left(G_{2}\right)+4 n_{2}^{3} M_{1}\left(G_{1}\right)+4 e_{1} n_{2}^{3}+4 e_{1} n_{2}^{2}(k-1)+8 n_{2} e_{1}(k-1) \sum_{i=1}^{n_{2}-1}\left(n_{2}-i\right)
$$


Proof. Let $d(u, v)=d_{G_{1}\left[G_{2}\right]_{R_{k}}}(u, v)$ be the degree of a vertex $(u, v)$ in the graph $G_{1}\left[G_{2}\right]_{R_{k}}$ :

$$
\begin{aligned}
& M_{1}\left(G_{1}\left[G_{2}\right]_{R_{k}}\right) \sum_{(u, v) \varepsilon V\left(G_{1}\left[G_{2}\right]_{R_{k}}\right)} d^{2}(u, v)=\sum_{\left(u_{1}, v_{1}\right)\left(u_{2}, v_{2}\right) \varepsilon E\left(G_{1}\left[G_{2}\right]_{R_{k}}\right)}\left[d\left(u_{1}, v_{1}\right)+d\left(u_{2}, v_{2}\right)\right] \\
& =\sum_{u_{1}=u_{2}=u \varepsilon V\left(G_{1}\right)} \sum_{v_{1} v_{2} \varepsilon E\left(G_{2}\right)}\left[d\left(u, v_{1}\right)+d\left(u, v_{2}\right)\right]+\sum_{v_{1} \varepsilon V\left(G_{2}\right)} \sum_{v_{2} \varepsilon V\left(G_{2}\right)} \sum_{u_{1} u_{2} \varepsilon E\left(R_{k}\left(G_{2}\right)\right)} \\
& \cdot\left[d\left(u_{1}, v_{1}\right)+d\left(u_{2}, v_{2}\right)\right] \\
& +\sum_{v_{1}=v_{2}=v \varepsilon V\left(G_{2}\right)} \sum_{\substack{u_{1} u_{2} \varepsilon E\left(R_{k}\left(G_{1}\right)\right) \\
u_{1} u_{2} \varepsilon v\left[R_{k}\left(G_{1}\right)-V\left(G_{1}\right)\right]}}\left[d\left(u_{1}, v_{1}\right)+d\left(u_{2}, v_{2}\right)\right] \\
& +\sum_{v_{1} \varepsilon V\left(G_{2}\right)} \sum_{v_{2} \varepsilon V\left(G_{2}\right)} \sum_{u_{1} u_{2} \varepsilon E\left(R_{k}\left(G_{2}\right)\right)}\left[d\left(u_{1}, v_{1}\right)+d\left(u_{2}, v_{2}\right)\right] \\
& =\sum 1+\sum 2+\sum 3+\sum 4
\end{aligned}
$$

Now, first, we calculate

$$
\begin{aligned}
& \sum 1=\sum_{u_{1}=u_{2}=u \varepsilon V} \sum_{\left(G_{1}\right)}\left[d\left(u, v_{1}\right)+d\left(u, v_{2}\right)\right] \\
& =\sum_{u \varepsilon V\left(G_{1}\right)} \sum_{v_{1} v_{2} \varepsilon E\left(G_{2}\right)}\left[n_{2} d_{R_{k G_{1}}} u+d_{G_{2}} v_{1}+n_{2} d_{R_{k G_{1}} u}+d_{G_{2}} v_{2}\right] \\
& =\sum_{u \varepsilon V\left(G_{1}\right)} \sum_{v_{1} v_{2} \varepsilon E\left(G_{2}\right)}\left[2 n_{2} d_{R_{k G_{1}}} u+d_{G_{2}} v_{1}+d_{G_{2}} v_{2}\right] \\
& =\sum_{u \varepsilon V\left(G_{1}\right)} \sum_{v_{1} v_{2} \varepsilon E\left(G_{2}\right)}\left[4 n_{2} d_{G_{1}} u+d_{G_{2}} v_{1}+d_{G_{2}} v_{2}\right] \\
& =4 n_{2} \sum_{v_{1} v_{2} \varepsilon E\left(G_{2}\right)}\left[\sum_{u \varepsilon V\left(G_{1}\right)}\left[d_{G_{1} u}\right]+\sum_{u \varepsilon V\left(G_{1}\right)} \sum_{v_{1} v_{2} \varepsilon E\left(G_{2}\right)}\left[d_{G_{2}} v_{1}+d_{G_{2}} v_{2}\right]\right] \\
& =4 n_{2} \sum_{v_{1} v_{2} \varepsilon E\left(G_{2}\right)}\left(e_{1}\right)+\sum_{u \varepsilon V\left(G_{1}\right)} M_{1}\left(G_{2}\right) \\
& =4 n_{2}\left(2 e_{2}\right)\left(e_{1}\right)+n_{1} M_{1}\left(G_{2}\right)=8 n_{2} e_{1} e_{2}+n_{1} M_{1}\left(G_{2}\right), \\
& \sum 2=\sum_{v_{1} \varepsilon V\left(G_{2}\right)} \sum_{v_{2} \varepsilon V\left(G_{2}\right)} \sum_{\substack{u_{1} u_{2} \varepsilon E\left(G_{1}\right) \\
u_{1} \varepsilon v\left(G_{1}\right) u_{2} \varepsilon v\left[R_{k}\left(G_{1}\right)-V\left(G_{1}\right)\right]}}\left[d\left(u_{1}, v_{1}\right)+d\left(u_{2}, v_{2}\right)\right] \\
& +\sum_{v_{1} \varepsilon V\left(G_{2}\right)} \sum_{v_{2} \varepsilon V\left(G_{2}\right)} \sum_{\substack{u_{1} u_{2} \varepsilon E\left[R_{k}\left(G_{1}\right)\right] \\
u_{1} \varepsilon v\left(G_{1}\right) u_{2} \varepsilon v\left[R_{k}\left(G_{1}\right)-V\left(G_{1}\right)\right]}}\left[d\left(u_{1}, v_{1}\right)+d\left(u_{2}, v_{2}\right)\right] \\
& +\sum_{v_{1}=v_{2}=v \varepsilon V\left(G_{2}\right)} \sum_{\substack{u_{1} u_{2} \varepsilon E\left(R_{k}\left(G_{1}\right)\right) \\
u_{1} u_{2} \varepsilon \nabla\left[R_{k}\left(G_{1}\right)-V\left(G_{1}\right)\right]}}\left[d\left(u_{1}, v_{1}\right)+d\left(u_{2}, v_{2}\right)\right]
\end{aligned}
$$




$$
\begin{aligned}
& \sum 2=\sum 2^{\prime}+\sum 2^{\prime \prime} \\
& \sum 2^{\prime}=\sum_{v_{1} \varepsilon V\left(G_{2}\right)} \sum_{v_{2} \varepsilon V\left(G_{2}\right)} \sum_{u_{1} u_{2} \varepsilon E\left(G_{1}\right)} \quad\left[d\left(u_{1}, v_{1}\right)+d\left(u_{2}, v_{2}\right)\right] \\
& u_{1} \varepsilon v\left(G_{1}\right) u_{2} \varepsilon v\left[R_{k}\left(G_{1}\right)-V\left(G_{1}\right)\right] \\
& =\sum_{v_{1} \varepsilon V\left(G_{2}\right)} \sum_{v_{2} \varepsilon V\left(G_{2}\right)} \sum_{u_{1} u_{2} \varepsilon E\left(G_{1}\right)}\left(n_{2} d_{R_{k G_{1}}} u_{1}+d_{G_{2}} v_{1}\right)+\left(n_{2} d_{R_{k G_{1}}} u_{2}+d_{G_{2}} v_{2}\right) \\
& =\sum_{v_{1} \varepsilon V\left(G_{2}\right)} \sum_{v_{2} \varepsilon V\left(G_{2}\right)} \sum_{u_{1} u_{2} \varepsilon E\left(G_{1}\right)} n_{2}\left(d_{R_{k G_{1}}} u_{1}+d_{R_{k G_{1}}} u_{2}\right)+\left(d_{G_{2}} v_{1}+d_{G_{2}} v_{2}\right) \\
& =\sum_{v_{1} \varepsilon V\left(G_{2}\right)} \sum_{v_{2} \varepsilon V\left(G_{2}\right)} \sum_{u_{1} u_{2} \varepsilon E\left(G_{1}\right)} 2 n_{2}\left(d_{G_{1}} u_{1}+d_{G_{1}} u_{2}\right)+\left(d_{G_{2}} v_{1}+d_{G_{2}} v_{2}\right) \\
& =\sum_{v_{1} \varepsilon V\left(G_{2}\right)} \sum_{v_{2} \varepsilon V\left(G_{2}\right)} \sum_{u_{1} u_{2} \varepsilon E\left(G_{1}\right)} 2 n_{2} M_{1}\left(G_{1}\right)+2 e_{2} e_{1} n_{2}+2 e_{1} e_{2} n_{2} \\
& =n_{2} n_{2} 2 n_{2} M_{1}\left(G_{1}\right)+4 e_{1} e_{2} n_{2}=2 n_{2}^{3} M_{1}\left(G_{1}\right)+4 e_{1} e_{2} n_{2}, \\
& \sum 2^{\prime \prime}=\sum_{v_{1} \varepsilon V\left(G_{2}\right)} \sum_{v_{2} \varepsilon V\left(G_{2}\right)} \sum_{u_{1} u_{2} \varepsilon E\left[R_{k}\left(G_{1}\right)\right]} \quad\left[d\left(u_{1}, v_{1}\right)+d\left(u_{2}, v_{2}\right)\right] \\
& u_{1} \varepsilon v\left(G_{1}\right) u_{2} \varepsilon v\left[R_{k}\left(G_{1}\right)-V\left(G_{1}\right)\right] \\
& =\sum_{v_{1} \varepsilon V\left(G_{2}\right)} \sum_{v_{2} \varepsilon V\left(G_{2}\right)} \sum_{u_{1} u_{2} \varepsilon E\left[R_{k}\left(G_{1}\right)\right]} \quad\left[n_{2} d_{R_{k G_{1}}} u_{1}+d_{G_{2}} v_{1}+n_{2} d_{R_{k G_{1}}} u_{2}\right] \\
& u_{1} \varepsilon v\left(G_{1}\right) u_{2} \varepsilon v\left[R_{k}\left(G_{1}\right)-v\left(G_{1}\right)\right] \\
& =\sum_{v_{1} \varepsilon V\left(G_{2}\right)} \sum_{v_{2} \varepsilon V\left(G_{2}\right)} \sum_{\substack{u_{1} u_{2} \varepsilon E\left[R_{k}\left(G_{1}\right)\right] \\
u_{1} \varepsilon v\left(G_{1}\right) u_{2} \varepsilon v\left[R_{k}\left(G_{1}\right)-V\left(G_{1}\right)\right]}}\left[2 n_{2} d_{G_{1}} u_{1}+d_{G_{2}} v_{1}+2 n_{2}\right] \\
& =n_{1} n_{2} 2 n_{2} \sum_{\substack{u_{1} u_{2} \varepsilon E\left[R_{k}\left(G_{1}\right)\right] \\
u_{1} \varepsilon v\left(G_{1}\right) u_{2} \varepsilon v\left[R_{k}\left(G_{1}\right)-V\left(G_{1}\right)\right]}} d_{G_{1}}\left(u_{1}\right)+\sum_{v_{1} \varepsilon V\left(G_{2}\right)} \sum_{\substack{v_{2} \varepsilon V\left(R_{k 2}\right) \\
u_{1} u_{2} \varepsilon E\left[R_{k}\left(G_{1}\right)\right] \\
u_{1} \varepsilon v\left(G_{1}\right) u_{2} \varepsilon v\left[R_{k}\left(G_{1}\right)-V\left(G_{1}\right)\right]}}\left[d_{G_{2}} v_{1}+2 n_{2}\right] \\
& =2 n_{2}^{3} \sum_{u_{1} \varepsilon v\left(G_{1}\right)} d_{G_{1}}^{2} u_{1} \sum_{v_{1} \varepsilon V\left(G_{2}\right)} \sum_{v_{2} \varepsilon V\left(G_{2}\right)} \sum_{u_{1} \varepsilon v\left(G_{1}\right)} d_{G_{1}} u_{1}\left[d_{G_{2}} v_{1}+2 n_{2}\right] \\
& =2 n_{2}^{3} M_{1}\left(G_{1}\right)+2 e_{1} \sum_{v_{1} \varepsilon V\left(G_{2}\right)} \sum_{v_{2} \varepsilon V\left(G_{2}\right)}\left[d_{G_{2}} v_{1}+2 n_{2}\right] \\
& =2 n_{2}^{3} M_{1}\left(G_{1}\right)+2 e_{1}\left[2 e_{2} n_{2}+2 n_{2} n_{2} n_{2}\right]=2 n_{2}^{3} M_{1}\left(G_{1}\right)+4 e_{1} e_{2} n_{2}+4 e_{1} n_{2}^{3}, \\
& \sum 3=\sum_{v_{1}=v_{2}=v \varepsilon V} \sum_{\left(G_{2}\right)} \sum_{u_{1} u_{2} \varepsilon E\left(R_{k}\left(G_{1}\right)\right)}\left[d\left(u_{1}, v_{1}\right)+d\left(u_{2}, v_{2}\right)\right] \\
& u_{1} u_{2} \varepsilon \nabla\left[R_{k}\left(G_{1}\right)-V\left(G_{1}\right)\right] \\
& =n_{2}(k-1) e_{1}\left[2 n_{2}+2 n_{2}\right]=4 e_{1} n_{2}^{2}(k-1), \\
& \sum 4=\sum_{v_{1} \varepsilon V\left(G_{2}\right)} \sum_{v_{2} \varepsilon V\left(G_{2}\right)} \sum_{u_{1} u_{2} \varepsilon E\left(R_{k}\left(G_{1}\right)\right)}\left[d\left(u_{1}, v_{1}\right)+d\left(u_{2}, v_{2}\right)\right] \\
& =\left[2 n_{2}+2 n_{2}\right] \sum_{i=1}^{n_{2}-1}\left(2 n_{2}-2 i\right)(k-2) e_{1}+\left[2 n_{2}+2 n_{2}\right] \sum_{i=1}^{n_{2}-1}\left(2 n_{2}-2 i\right) e_{1} \\
& =8 n_{2} \sum_{i=1}^{n_{2}-1}\left(n_{2}-i\right)(k-2) e_{1}+8 n_{2} \sum_{i=1}^{n_{2}-1}\left(n_{2}-i\right) e_{1}
\end{aligned}
$$


So, the final result is

$$
\begin{aligned}
= & 16 n_{2} e_{1} e_{2}+n_{1} M_{1}\left(G_{2}\right)+4 n_{2}^{3} M_{1}\left(G_{1}\right)+4 e_{1} n_{2}^{3} \\
& +4 e_{1} n_{2}^{2}(k-1)+8 n_{2} e_{1}(k-1) \sum_{i=1}^{n_{2}-1} .
\end{aligned}
$$

Theorem 4. Let $G_{1}$ and $G_{2}$ be two networks; then, for some integer $k$, the second Zagreb index of $R_{k}$-sum network is

$$
\begin{aligned}
M_{2}\left(G_{1}\left[G_{2}\right]_{R_{k}}\right)= & 8 n_{2}^{2} e_{2} M_{1}\left(G_{1}\right)+4 e_{1} n_{2} M_{1}\left(G_{2}\right)+n_{1} M_{2}\left(G_{2}\right)+4 n_{2}^{4} M_{2}\left(G_{1}\right)+4 n_{2}^{4} M_{1}\left(G_{2}\right)+8 n_{2}^{2} e_{1} e_{2}+4 e_{1} e_{2}^{2} \\
& +4 e_{1} n_{3}^{2}(k-1)+8 n_{2}^{2} e_{1}(k-1) \sum_{i=1}^{n_{2}-1}\left(n_{2}-i\right) .
\end{aligned}
$$

Proof. Let $d(u, v)=d_{G_{1}\left[G_{2}\right]_{R_{k}}}(u, v)$ be the degree of a vertex $(u, v)$ in the network $G_{1}\left[G_{2}\right]_{R_{k}}$ :

$$
\begin{aligned}
M_{1}\left(G_{1}\left[G_{2}\right]_{R_{k}}\right) \sum_{(u, v) \varepsilon V\left(G_{1}\left[G_{2}\right]_{R_{k}}\right)} d^{2}(u, v)= & \sum_{\left(u_{1}, v_{1}\right)\left(u_{2}, v_{2}\right) \varepsilon E\left(G_{1}\left[G_{2}\right]_{R_{k}}\right)}\left[d\left(u_{1}, v_{1}\right) d\left(u_{2}, v_{2}\right)\right] \\
= & \sum_{u_{1}=u_{2}=u \varepsilon V\left(G_{1}\right)} \sum_{v_{1} v_{2} \varepsilon E\left(G_{2}\right)}\left[d\left(u, v_{1}\right) d\left(u, v_{2}\right)\right]+\sum_{v_{1} \varepsilon V\left(G_{2}\right)} \sum_{v_{2} \varepsilon V\left(G_{2}\right) u_{1} u_{2} \varepsilon E\left(R_{k}\left(G_{2}\right)\right)} \sum^{\cdot\left[d\left(u_{1}, v_{1}\right) d\left(u_{2}, v_{2}\right)\right]} \\
& +\sum_{v_{1}=v_{2}=v \varepsilon V\left(G_{2}\right)} \sum_{u_{1} u_{2} \varepsilon E\left(R_{k}\left(G_{1}\right)\right)}^{u_{1} u_{2} \varepsilon \nabla\left[R_{k}\left(G_{1}\right)-V\left(G_{1}\right)\right]}\left[d\left(u_{1}, v_{1}\right)+d\left(u_{2}, v_{2}\right)\right] \\
& +\sum_{v_{1} \varepsilon V\left(G_{2}\right)} \sum_{v_{2} \varepsilon V\left(G_{2}\right)}\left[d \left(u_{1} u_{2} \varepsilon E\left(R_{k}\left(G_{2}\right)\right)\right.\right. \\
= & \sum 1+\sum 2+\sum 3+\sum 4 .
\end{aligned}
$$

Now, first, we calculate

$$
\begin{aligned}
\sum 1 & =\sum_{u_{1}=u_{2}=u \varepsilon V} \sum_{\left(G_{1}\right)}\left[d\left(u, v_{1}\right) d\left(u, v_{2}\right)\right] \\
& =\sum_{u \varepsilon V\left(G_{1}\right)} \sum_{v_{1} v_{2} \varepsilon E\left(G_{2}\right)}\left[n_{2} d_{R_{k G_{1}}} u+d_{G_{2}} v_{1}\right]\left[n_{2} d_{R_{k G_{1}} u}+d_{G_{2}} v_{2}\right] \\
& =\sum_{u \varepsilon V\left(G_{1}\right)} \sum_{v_{1} v_{2} \varepsilon E\left(G_{2}\right)}\left[2 n_{2} d_{G_{1}} u+d_{G_{2}} v_{1}\right]\left[2 n_{2} d_{G_{1} u}+d_{G_{2}} v_{2}\right] \\
& =\sum_{u \varepsilon V\left(G_{1}\right)} \sum_{v_{1} v_{2} \varepsilon E\left(G_{2}\right)}\left[4 n_{2}^{2} d_{G_{1}}^{2} u+2 n_{2} d_{G_{1} u}\left[d_{G_{2}} v_{1}+d_{G_{2}} v_{2}\right]\right] \\
& =4 e_{2} n_{2}^{2} M_{1}\left(G_{1}\right)+4 e_{1} n_{2} M_{1}\left(G_{2}\right)+n_{1} M_{2}\left(G_{2}\right),
\end{aligned}
$$




$$
\begin{aligned}
& \sum 2=\sum_{v_{1} \varepsilon V\left(G_{2}\right)} \sum_{v_{2} \varepsilon V\left(G_{2}\right)} \sum_{\substack{u_{1} u_{2} \varepsilon E\left(G_{1}\right) \\
u_{1} v v\left(G_{1}\right) u_{2} \varepsilon v\left[R_{k}\left(G_{1}\right)-V\left(G_{1}\right)\right]}}\left[d\left(u_{1}, v_{1}\right) d\left(u_{2}, v_{2}\right)\right] \\
& +\sum_{v_{1} \varepsilon V\left(G_{2}\right)} \sum_{v_{2} \varepsilon V\left(G_{2}\right)} \sum_{\substack{u_{1} u_{2} \varepsilon\left[\left[R_{k}\left(G_{1}\right)\right] \\
u_{1} \varepsilon v V\left(G_{1}\right) u_{2} \varepsilon v\left[R_{k}\left(G_{1}\right)-V\left(G_{1}\right)\right]\right.}}\left[d\left(u_{1} v_{1}\right) d\left(u_{2} v_{2}\right)\right] \\
& \sum 2=\sum 2^{\prime}+\sum 2^{\prime \prime} \\
& \sum 2^{\prime}=\sum_{v_{1} \& V\left(G_{2}\right)} \sum_{v_{2} \varepsilon V\left(G_{2}\right)} \sum_{u_{1} u_{2} E E\left(R_{k}\left(G_{1}\right)\right)}\left[d\left(u_{1}, v_{1}\right) \cdot d\left(u_{2}, v_{2}\right)\right] \\
& =\sum_{v_{1} \varepsilon V\left(G_{2}\right)} \sum_{v_{2} \varepsilon V\left(G_{2}\right)} \sum_{u_{1} u_{2} E E\left(G_{1}\right)}\left[d\left(u_{1}, v_{1}\right) d\left(u_{2}, v_{2}\right)\right] \\
& =\sum_{v_{1} \varepsilon V\left(G_{2}\right)} \sum_{v_{2} E V\left(G_{2}\right)} \sum_{u_{1} u_{2} E E\left(G_{1}\right)}\left[n_{2} d_{R_{K G_{1}}} u_{1}+d_{G_{2}} v_{1}\right]\left[n_{2} d_{R_{K G_{1}}} u_{2}+d_{G_{2}} v_{2}\right] \\
& =\sum_{v_{1} \varepsilon V\left(G_{2}\right)} \sum_{v_{2} E V\left(G_{2}\right)} \sum_{u_{1} u_{2} E E\left(G_{1}\right)}\left[2 n_{2} d_{G_{1}} u_{1}+d_{G_{2}} v_{1}\right]\left[n_{2} d_{G_{1}} u_{2}+d_{G_{2}} v_{2}\right] \\
& =\sum_{v_{1} \varepsilon V\left(G_{2}\right)} \sum_{v_{2} \varepsilon V\left(G_{2}\right)} \sum_{u_{1} u_{2} \varepsilon E\left(G_{1}\right)}\left[4 n_{2}^{2} d_{G_{1}} u_{1} d_{G_{1}} u_{2}+2 n_{2} d_{G_{1}} u_{1} d_{G_{2}} v_{2}+2 n_{2} d_{G_{1}} u_{2} d_{G_{2}} v_{1}+d_{G_{2}} v_{1} d_{G_{2}} v_{2}\right] \\
& =4 n_{2}^{4} M_{2}\left(G_{1}\right)+4 e_{2} n_{2}^{2} \sum_{u_{1} u_{2} E\left(G_{1}\right)}\left[d_{G_{1}} u_{1}+d_{G_{1}} u_{2}\right]+4 e_{1} e_{2}^{2}=4 n_{2}^{4} M_{2}\left(G_{1}\right)+4 e_{2} n_{2}^{2} M_{1}\left(G_{1}\right)+4 e_{1} e_{2}^{2} \text {, }
\end{aligned}
$$$$
\sum 2^{\prime \prime}=\sum_{v_{1} \varepsilon V\left(G_{2}\right)} \sum_{v_{2} \varepsilon V\left(G_{2}\right)} \sum_{\substack{u_{1} u_{2} \varepsilon\left(G_{1}\right) \\ u_{1} v V\left(G_{1}\right) u_{2} \varepsilon v\left[R_{k}\left(G_{1}\right)-V\left(G_{1}\right)\right]}}\left[d\left(u_{1}, v_{1}\right) d\left(u_{2}, v_{2}\right)\right]
$$$$
=\sum_{v_{1} \varepsilon V\left(G_{2}\right)} \sum_{v_{2} \varepsilon V\left(G_{2}\right)} \sum_{u_{1} u_{2} \varepsilon E\left(G_{1}\right)}\left[d\left(u_{1}, v_{1}\right) d\left(u_{2}, v_{2}\right)\right]
$$$$
u_{1} \varepsilon v\left(G_{1}\right) u_{2} \varepsilon v\left[R_{k}\left(G_{1}\right)-V\left(G_{1}\right)\right]
$$$$
=\sum_{v_{1} E V\left(G_{2}\right)} \sum_{v_{2} \varepsilon V\left(G_{2}\right)} u_{u_{1} u_{2} E E\left(G_{1}\right)} \sum \quad\left[n_{2} d_{R_{k G_{1}}} u_{1}+d_{G_{2}} v_{1}\right]\left[n_{2} d_{R_{k G_{1}}} u_{2}\right]
$$$$
u_{1} \varepsilon v\left(G_{1}\right) u_{2} \varepsilon v\left[R_{k}\left(G_{1}\right)-V\left(G_{1}\right)\right]
$$$$
=\sum_{v_{1} \varepsilon V\left(G_{2}\right)} \sum_{v_{2} E V\left(G_{2}\right)} u_{u_{1} u_{2} E E\left(G_{1}\right)} \sum \quad\left[n_{2} d_{R_{k G_{1}}} u_{1}+d_{G_{2}} v_{1}\right]\left[2 n_{2}\right]
$$$$
u_{1} \varepsilon v\left(G_{1}\right) u_{2} \varepsilon v\left[R_{k}\left(G_{1}\right)-v\left(G_{1}\right)\right]
$$$$
=\sum_{v_{1} \varepsilon V\left(G_{2}\right)} \sum_{v_{2} \varepsilon V\left(G_{2}\right)} \sum_{u_{1} u_{2} \varepsilon E\left(R_{k}\left(G_{1}\right)\right)}\left[4 n_{2}^{2}\left[d_{G_{1}} u_{1}+2 n_{2} d_{G_{2}} v_{1}\right]\right]
$$$$
=4 n_{2}^{4} \sum_{u_{1} u_{2} \varepsilon E\left(N_{2}\left(G_{1}\right)\right)} d_{G_{1}} u_{1}+\sum_{v_{1} \varepsilon V\left(G_{2}\right)} \sum_{v_{2} \varepsilon V\left(G_{2}\right)} \sum_{u_{1} u_{2} E E\left(R_{k}\left(G_{1}\right)\right)}\left[2 n_{2} d_{G_{2}} v_{1}\right]
$$$$
=4 n_{2}^{4} \sum_{u_{1} \varepsilon V\left(G_{1}\right)} d_{G_{1}}^{2} u_{1}+4 e_{2} n_{2}^{2} \sum_{u_{1} \varepsilon V\left(G_{1}\right)} d_{G_{1}} u_{1}=4 n_{2}^{4} M_{1}\left(G_{2}\right)+8 e_{1} e_{2} n_{2}^{2} \text {, }
$$

$$
\sum 3=\sum_{v_{1}=v_{2}=v \varepsilon V\left(G_{2}\right)} \sum_{\substack{\left.u_{1} u_{2} \varepsilon E\left(R_{k}\right)\left(G_{1}\right)\right) \\ u_{1} u_{2} E V\left[R_{k}\left(G_{1}\right)-V\left(G_{1}\right)\right]}}\left[d\left(u_{1}, v_{1}\right) d\left(u_{2}, v_{2}\right)\right],
$$


TABLE 1: Numerical comparison for generalized network $G\left(P_{n}\left[P_{m}\right]_{T_{k}}\right)$ for $k=1$.

\begin{tabular}{|c|c|c|c|c|}
\hline$[m, n]$ & $M_{1}(G)\left(P_{n}\left[P_{m}\right]_{S_{k}}\right)$ & $M_{2}(G)\left(P_{n}\left[P_{m}\right]_{S_{k}}\right)$ & $M_{1}(G)\left(P_{n}\left[P_{m}\right]_{R_{k}}\right)$ & $M_{2}(G)\left(P_{n}\left[P_{m}\right]_{R_{k}}\right)$ \\
\hline$(5,5)$ & 4460 & 22920 & 10350 & 80636 \\
\hline$(5,6)$ & 5634 & 29312 & 13184 & 104952 \\
\hline$(5,7)$ & 6808 & 35704 & 16018 & 129268 \\
\hline$(5,8)$ & 7982 & 42096 & 18852 & 153584 \\
\hline$(5,9)$ & 9156 & 48488 & 21686 & 177900 \\
\hline$(5,10)$ & 10330 & 54880 & 24520 & 202216 \\
\hline$(6,5)$ & 7530 & 45752 & 17562 & 162672 \\
\hline$(6,6)$ & 9516 & 58560 & 22380 & 211844 \\
\hline$(6,7)$ & 11502 & 71368 & 27198 & 261016 \\
\hline$(6,8)$ & 13488 & 841767 & 32016 & 310188 \\
\hline$(6,9)$ & 15474 & 96984 & 36834 & 359360 \\
\hline$(6,10)$ & 17460 & 109792 & 41652 & 408532 \\
\hline$(7,5)$ & 11744 & 823647 & 27494 & 294900 \\
\hline$(7,6)$ & 14846 & 105484 & 35048 & 384216 \\
\hline$(7,7)$ & 17948 & 128604 & 42602 & 473532 \\
\hline$(7,8)$ & 21050 & 151724 & 50156 & 562848 \\
\hline$(7,9)$ & 24152 & 174844 & 57710 & 652164 \\
\hline$(7,10)$ & 27254 & 197964 & 65264 & 741480 \\
\hline$(8,5)$ & 17282 & 137400 & 40578 & 494408 \\
\hline$(8,6)$ & 21852 & 176048 & 51740 & 644388 \\
\hline$(8,7)$ & 26422 & 214696 & 62902 & 794368 \\
\hline$(8,8)$ & 30992 & 253344 & 74064 & 944348 \\
\hline$(8,9)$ & 35562 & 291992 & 85226 & 1094328 \\
\hline$(8,10)$ & 40132 & 330640 & 96388 & 1244308 \\
\hline$(9,5)$ & 24324 & 216176 & 57246 & 780780 \\
\hline$(9,6)$ & 30762 & 277080 & 73008 & 1017944 \\
\hline$(9,7)$ & 37200 & 337984 & 88770 & 1255108 \\
\hline$(9,8)$ & 43638 & 398888 & 104532 & 1492272 \\
\hline$(9,9)$ & 50076 & 459792 & 120294 & 1729436 \\
\hline$(9,10)$ & 56514 & 520696 & 136056 & 1966600 \\
\hline$(10,5)$ & 33050 & 324680 & 77930 & 1176096 \\
\hline$(10,6)$ & 41804 & 416272 & 99404 & 1533732 \\
\hline$(10,7)$ & 50558 & 507864 & 120878 & 1891368 \\
\hline$(10,8)$ & 59312 & 599456 & 142352 & 2249004 \\
\hline$(10,9)$ & 68066 & 691048 & 163826 & 2606640 \\
\hline$(10,10)$ & 76820 & 782640 & 185300 & 2964276 \\
\hline
\end{tabular}

$$
\begin{aligned}
\sum_{v_{1}=v_{2}=v \varepsilon V} \sum_{\left.G_{2}\right)}\left[d\left(u_{1}, v_{1}\right) d\left(u_{2}, v_{2}\right)\right] & =n_{2}(k-1) e_{1}\left[2 n_{2} \cdot 2 n_{2}\right]=4 e_{1} n_{2}^{3}(k-1) \\
\sum 4 & =\sum_{v_{1} \varepsilon V\left(G_{2}\right)} \sum_{v_{2} \varepsilon V\left(G_{2}\right)} \sum_{u_{1} u_{2} \varepsilon E\left(R_{k}\left(G_{1}\right)\right)}\left[d\left(u_{1}, v_{1}\right) d\left(u_{2}, v_{2}\right)\right] \\
& =\left[2 n_{2} \times 2 n_{2}\right] \sum_{i=1}^{n_{2}-1}\left(2 n_{2}-2 i\right)(k-2) e_{1}+\left[2 n_{2} \times 2 n_{2}\right] \sum_{i=1}^{n_{2}-1}\left(2 n_{2}-2 i\right) e_{1} \\
& =8 n_{2}^{2} \sum_{i=1}^{n_{2}-1}\left(n_{2}-i\right)(k-2) e_{1}+8 n_{2}^{2} \sum_{i=1}^{n_{2}-1}\left(n_{2}-i\right) e_{1}=8 n_{2}^{2} e_{1}(k-1) \sum_{i=1}^{n_{2}-1}\left(n_{2}-i\right) .
\end{aligned}
$$

So, the final result is

$$
\begin{aligned}
= & 8 n_{2}^{2} e_{2} M_{1}\left(G_{1}\right)+4 e_{1} n_{2} M_{1}\left(G_{2}\right)+n_{1} M_{2}\left(G_{2}\right) \\
& +4 n_{2}^{4} M_{2}\left(G_{1}\right)+4 n_{2}^{4} M_{1}\left(G_{2}\right)+8 n_{2}^{2} e_{1} e_{2}+4 e_{1} e_{2}^{2} \\
& +4 e_{1} n_{3}^{2}(k-1) \\
& +8 n_{2}^{2} e_{1}(k-1) \sum_{i=1}^{n_{2}-1}\left(n_{2}-i\right) .
\end{aligned}
$$

\section{Conclusion}

Let $G_{1}=P_{n}$ and $G_{2}=P_{m}$ be two networks; then, we have $n_{1}=\left|V\left(G_{1}\right)\right|=n, n_{2}=\left|V\left(G_{2}\right)\right|=m, e_{1}=\left|E\left(G_{1}\right)\right|=n-1$, $e_{2}=\left|E\left(G_{1}\right)\right|=m-1, M_{1}\left(G_{1}\right)=4 n-6, M_{1}\left(G_{2}\right)=4 m-6$, $M_{2}\left(G_{1}\right)=4(n-2)$, and $M_{2}\left(G_{2}\right)=4(m-2)$.

Applying these values on the above derived results in Theorems 1-4, we obtain Tables 1-4. The graphical 
TABLE 2: Numerical comparison for generalized networks $G\left(P_{n}\left[P_{m}\right]_{N_{k}}\right)$ for $k=2$.

\begin{tabular}{|c|c|c|c|c|}
\hline$[m, n]$ & $M_{1}(G)\left(P_{n}\left[P_{m}\right]_{S_{k}}\right)$ & $M_{2}(G)\left(P_{n}\left[P_{m}\right]_{S_{k}}\right)$ & $M_{1}(G)\left(P_{n}\left[P_{m}\right]_{R_{k}}\right)$ & $M_{2}(G)\left(P_{n}\left[P_{m}\right]_{R_{k}}\right)$ \\
\hline$(5,5)$ & 6660 & 31120 & 12350 & 103136 \\
\hline$(5,6)$ & 8374 & 39572 & 15684 & 129952 \\
\hline$(5,7)$ & 10088 & 48024 & 19018 & 156768 \\
\hline$(5,8)$ & 11802 & 56476 & 22352 & 183584 \\
\hline$(5,9)$ & 13516 & 64928 & 25686 & 210400 \\
\hline$(5,10)$ & 15230 & 73380 & 29020 & 237216 \\
\hline$(6,5)$ & 11226 & 63368 & 21018 & 209328 \\
\hline$(6,6)$ & 14124 & 80592 & 26700 & 263684 \\
\hline$(6,7)$ & 17022 & 97816 & 32382 & 318040 \\
\hline$(6,8)$ & 19920 & 115040 & 38064 & 372396 \\
\hline$(6,9)$ & 22818 & 132264 & 43746 & 426752 \\
\hline$(6,10)$ & 25716 & 149488 & 49428 & 481108 \\
\hline$(7,5)$ & 17512 & 115796 & 32982 & 381336 \\
\hline$(7,6)$ & 22042 & 147288 & 41908 & 480256 \\
\hline$(7,7)$ & 26572 & 178780 & 50834 & 579176 \\
\hline$(7,8)$ & 31102 & 210272 & 59760 & 678096 \\
\hline$(7,9)$ & 35632 & 241764 & 68686 & 777016 \\
\hline$(7,10)$ & 40162 & 273256 & 77612 & 875936 \\
\hline$(8,5)$ & 25794 & 195448 & 48770 & 641864 \\
\hline$(8,6)$ & 32476 & 248624 & 61980 & 808228 \\
\hline$(8,7)$ & 39158 & 301800 & 75190 & 974592 \\
\hline$(8,8)$ & 45840 & 354976 & 88400 & 1140956 \\
\hline$(8,9)$ & 52522 & 408152 & 101610 & 1307320 \\
\hline$(8,10)$ & 59204 & 461328 & 114820 & 1473684 \\
\hline$(9,5)$ & 36348 & 310424 & 68910 & 1016976 \\
\hline$(9,6)$ & 45774 & 394908 & 87588 & 1280384 \\
\hline$(9,7)$ & 55200 & 479392 & 106266 & 1543792 \\
\hline$(9,8)$ & 64626 & 563876 & 124944 & 1807200 \\
\hline$(9,9)$ & 74052 & 648360 & 143622 & 2070608 \\
\hline$(9,10)$ & 83478 & 732844 & 162300 & 2334016 \\
\hline$(10,5)$ & 49450 & 469880 & 93930 & 1536096 \\
\hline$(10,6)$ & 62284 & 597792 & 119404 & 1933732 \\
\hline$(10,7)$ & 75118 & 725704 & 144878 & 2331368 \\
\hline$(10,8)$ & 87952 & 853616 & 170352 & 2729004 \\
\hline$(10,9)$ & 100786 & 981528 & 195826 & 3126640 \\
\hline$(10,10)$ & 113620 & 1109440 & 221300 & 3524276 \\
\hline
\end{tabular}

TABle 3: Numerical comparison for generalized networks $G\left(P_{n}\left[P_{m}\right]_{N_{k}}\right)$ for $k=3$.

\begin{tabular}{|c|c|c|c|c|}
\hline$[m, n]$ & $M_{1}(G)\left(P_{n}\left[P_{m}\right]_{S_{k}}\right)$ & $M_{2}(G)\left(P_{n}\left[P_{m}\right]_{S_{k}}\right)$ & $M_{1}(G)\left(P_{n}\left[P_{m}\right]_{R_{k}}\right)$ & $M_{2}(G)\left(P_{n}\left[P_{m}\right]_{R_{k}}\right)$ \\
\hline$(5,5)$ & 8460 & 39520 & 14750 & 100636 \\
\hline$(5,6)$ & 10634 & 50072 & 18684 & 129952 \\
\hline$(5,7)$ & 12808 & 60624 & 22618 & 159268 \\
\hline$(5,8)$ & 14982 & 71176 & 26552 & 188584 \\
\hline$(5,9)$ & 17156 & 81728 & 30486 & 217900 \\
\hline$(5,10)$ & 19330 & 92280 & 34420 & 247216 \\
\hline$(6,5)$ & 14442 & 81224 & 25050 & 204144 \\
\hline$(6,6)$ & 18156 & 102912 & 31740 & 263684 \\
\hline$(6,7)$ & 21870 & 124600 & 38430 & 323224 \\
\hline$(6,8)$ & 25584 & 146288 & 45120 & 382764 \\
\hline$(6,9)$ & 29298 & 167976 & 51810 & 442304 \\
\hline$(6,10)$ & 33012 & 189664 & 58500 & 501844 \\
\hline$(7,5)$ & 22720 & 149508 & 39254 & 371732 \\
\hline$(7,6)$ & 28566 & 189428 & 49748 & 480256 \\
\hline$(7,7)$ & 34412 & 229348 & 60242 & 588780 \\
\hline$(7,8)$ & 40258 & 269268 & 70736 & 697304 \\
\hline$(7,9)$ & 46104 & 309188 & 81230 & 805828 \\
\hline$(7,10)$ & 51950 & 349108 & 91724 & 914352 \\
\hline
\end{tabular}


TABle 3: Continued.

\begin{tabular}{|c|c|c|c|c|}
\hline$[m, n]$ & $M_{1}(G)\left(P_{n}\left[P_{m}\right]_{S_{k}}\right)$ & $M_{2}(G)\left(P_{n}\left[P_{m}\right]_{S_{k}}\right)$ & $M_{1}(G)\left(P_{n}\left[P_{m}\right]_{R_{k}}\right)$ & $M_{2}(G)\left(P_{n}\left[P_{m}\right]_{R_{k}}\right)$ \\
\hline$(8,5)$ & 33666 & 253816 & 57986 & 625480 \\
\hline$(8,6)$ & 42332 & 321584 & 73500 & 808228 \\
\hline$(8,7)$ & 50998 & 389352 & 89014 & 990976 \\
\hline$(8,8)$ & 59664 & 457120 & 104528 & 1173724 \\
\hline$(8,9)$ & 68330 & 524888 & 120042 & 1356472 \\
\hline$(8,10)$ & 76996 & 592656 & 135556 & 1539220 \\
\hline$(9,5)$ & 47652 & 405032 & 81870 & 990732 \\
\hline$(9,6)$ & 59922 & 513168 & 103788 & 1280384 \\
\hline$(9,7)$ & 72192 & 621304 & 125706 & 1570036 \\
\hline$(9,8)$ & 84462 & 729440 & 147624 & 1859688 \\
\hline$(9,9)$ & 96732 & 837576 & 169542 & 2149340 \\
\hline$(9,10)$ & 109002 & 945712 & 191460 & 2438992 \\
\hline$(10,5)$ & 65050 & 615480 & 111530 & 1496096 \\
\hline$(10,6)$ & 81804 & 779792 & 141404 & 1933732 \\
\hline$(10,7)$ & 98558 & 944104 & 171278 & 2371368 \\
\hline$(10,8)$ & 115312 & 1108416 & 201152 & 2809004 \\
\hline$(10,9)$ & 132066 & 1272728 & 231026 & 3246640 \\
\hline$(10,10)$ & 148820 & 1437040 & 260900 & 3684276 \\
\hline
\end{tabular}

TABLE 4: Numerical comparison for generalized networks $G\left(P_{n}\left[P_{m}\right]_{N_{k}}\right)$ for $k=4$.

\begin{tabular}{|c|c|c|c|c|}
\hline$[m, n]$ & $M_{1}(G)\left(P_{n}\left[P_{m}\right]_{S_{k}}\right)$ & $M_{2}(G)\left(P_{n}\left[P_{m}\right]_{S_{k}}\right)$ & $M_{1}(G)\left(P_{n}\left[P_{m}\right]_{R_{k}}\right)$ & $M_{2}(G)\left(P_{n}\left[P_{m}\right]_{R_{k}}\right)$ \\
\hline$(5,5)$ & 10460 & 47920 & 16750 & 110636 \\
\hline$(5,6)$ & 13134 & 60572 & 21184 & 142452 \\
\hline$(5,7)$ & 15808 & 73224 & 25618 & 174268 \\
\hline$(5,8)$ & 18482 & 85876 & 30052 & 206084 \\
\hline$(5,9)$ & 21156 & 98528 & 34486 & 237900 \\
\hline$(5,10)$ & 23830 & 111180 & 38920 & 269716 \\
\hline$(6,5)$ & 17898 & 99080 & 28506 & 224880 \\
\hline$(6,6)$ & 22476 & 125232 & 36060 & 289604 \\
\hline$(6,7)$ & 27054 & 151384 & 43614 & 354328 \\
\hline$(6,8)$ & 31632 & 177536 & 51168 & 419052 \\
\hline$(6,9)$ & 36210 & 203688 & 58722 & 483776 \\
\hline$(6,10)$ & 40788 & 229840 & 66276 & 548500 \\
\hline$(7,5)$ & 28208 & 183220 & 44742 & 410148 \\
\hline$(7,6)$ & 35426 & 231568 & 56608 & 528276 \\
\hline$(7,7)$ & 42644 & 279916 & 68474 & 646404 \\
\hline$(7,8)$ & 49862 & 328264 & 80340 & 764532 \\
\hline$(7,9)$ & 57080 & 376612 & 92206 & 882660 \\
\hline$(7,10)$ & 64298 & 424960 & 104072 & 1000788 \\
\hline$(8,5)$ & 41858 & 312184 & 66178 & 691016 \\
\hline$(8,6)$ & 52572 & 394544 & 83740 & 890148 \\
\hline$(8,7)$ & 63286 & 476904 & 101302 & 1089280 \\
\hline$(8,8)$ & 74000 & 559264 & 118864 & 1288412 \\
\hline$(8,9)$ & 84714 & 641624 & 136426 & 1487544 \\
\hline$(8,10)$ & 95428 & 723984 & 153988 & 1686676 \\
\hline$(9,5)$ & 59316 & 499640 & 93534 & 1095708 \\
\hline$(9,6)$ & 74502 & 631428 & 118368 & 1411604 \\
\hline$(9,7)$ & 89688 & 763216 & 143202 & 1727500 \\
\hline$(9,8)$ & 104874 & 895004 & 168036 & 2043396 \\
\hline$(9,9)$ & 120060 & 1026792 & 192870 & 2359292 \\
\hline$(9,10)$ & 135246 & 1158580 & 217704 & 2675188 \\
\hline$(10,5)$ & 81050 & 761080 & 127530 & 1656096 \\
\hline$(10,6)$ & 101804 & 961792 & 161404 & 2133732 \\
\hline$(10,7)$ & 122558 & 1162504 & 195278 & 2611368 \\
\hline$(10,8)$ & 143312 & 1363216 & 229152 & 3089004 \\
\hline$(10,9)$ & 164066 & 1563928 & 263026 & 3566640 \\
\hline$(10,10)$ & 184820 & 1764640 & 296900 & 4044276 \\
\hline
\end{tabular}




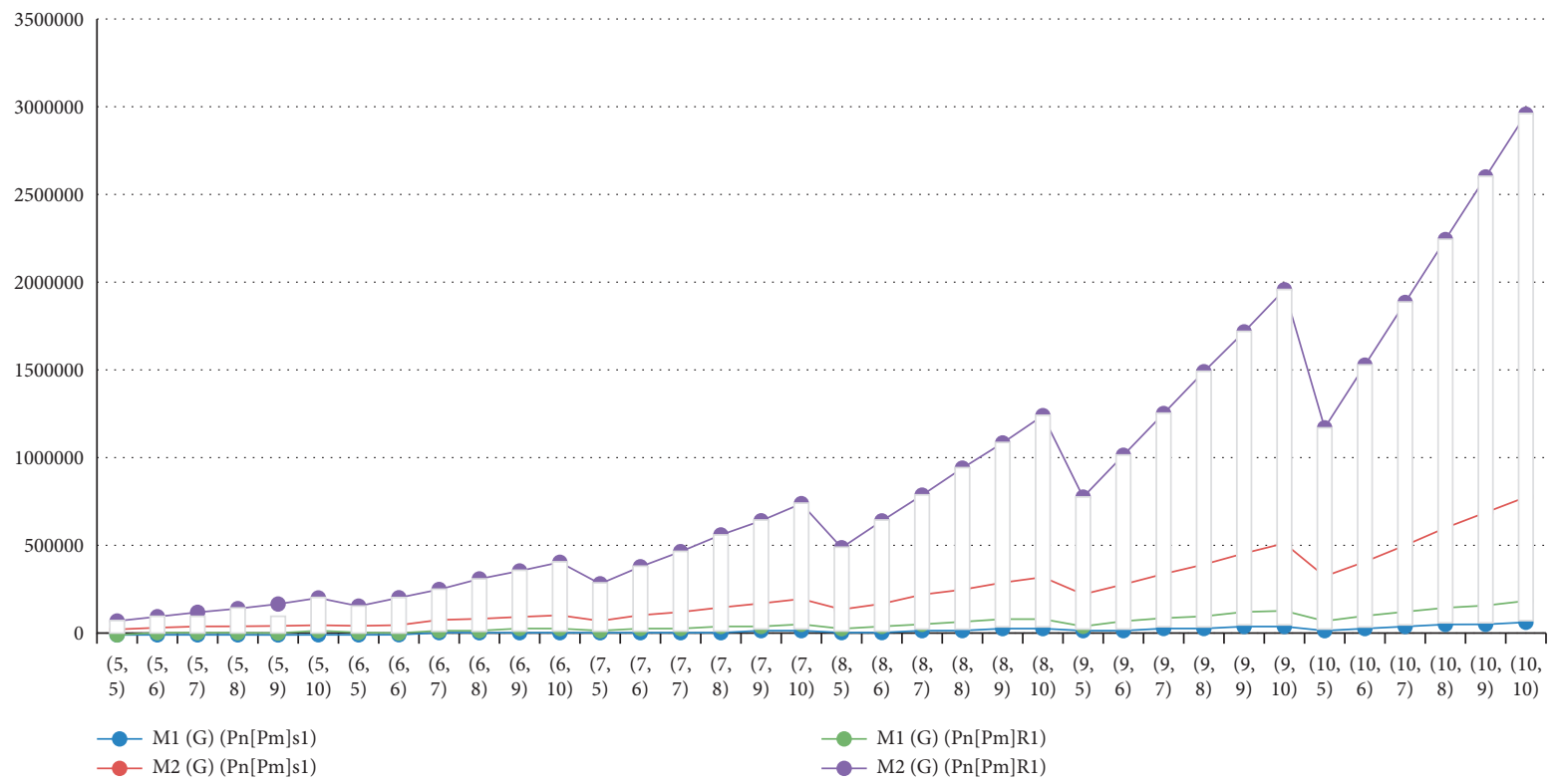

Figure 5: Numerical comparison of Zagreb indices of generalized subdivision and semitotal point operations with lexicographic product of the networks for $k=1$.

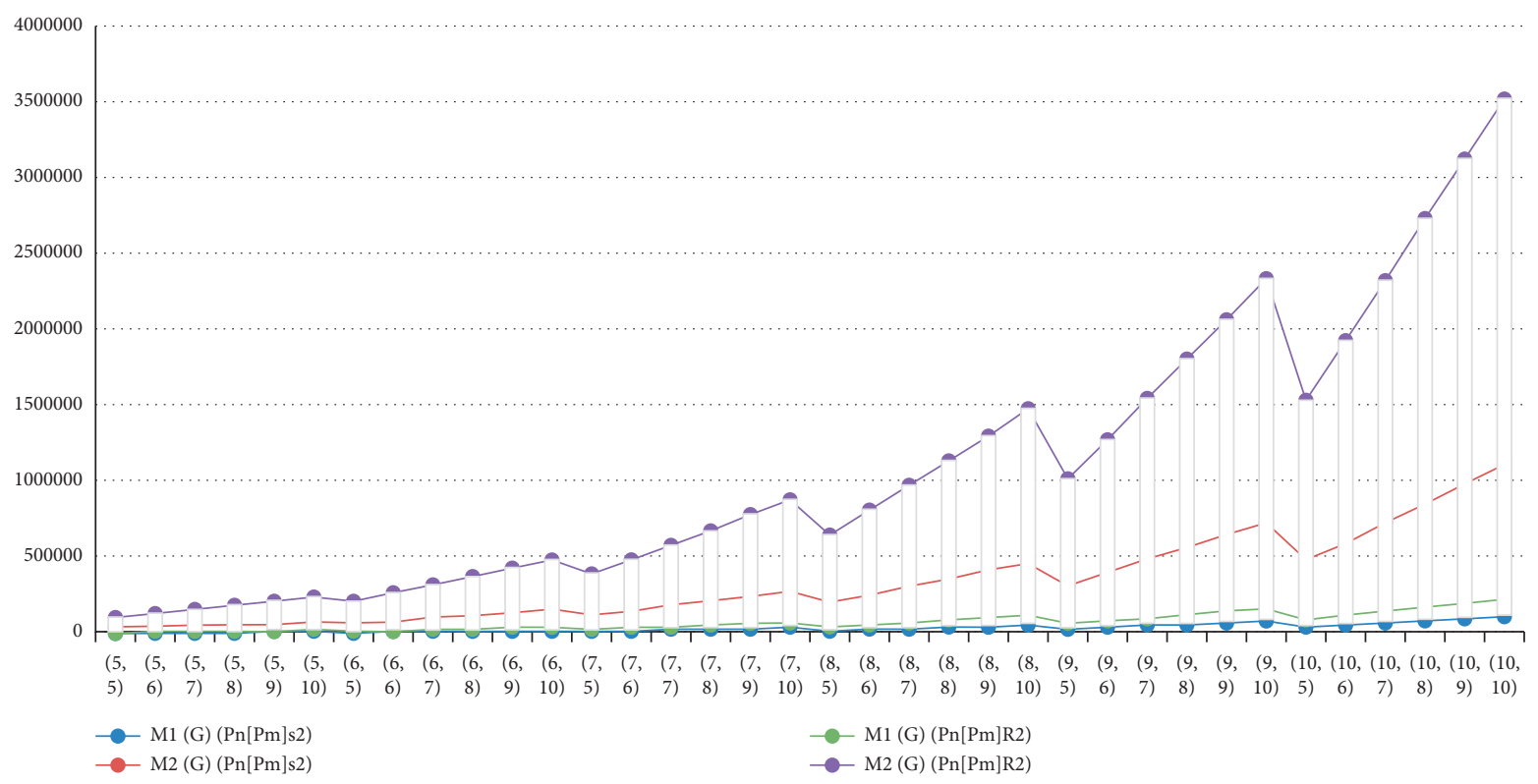

Figure 6: Numerical comparison of Zagreb indices of generalized subdivision and semitotal point operations with lexicographic product of the networks for $k=2$.

representations of the abovementioned results in tables are shown in Figures 5-8. In Figure 9, we summarize the results of generalized $T$-sum networks from $k=1$ to $k=4$.

For $k=1$,

(a) $G_{1} \quad\left(P_{n}\left[P_{m}\right] S\right)=8 m^{3} n-10 m^{3}+8 m^{2} n-8 m^{2}-$ $4 m n+8 m-6 n$

(b) $G_{2}\left(P_{n} \quad\left[P_{m}\right] S\right)=8 m^{4} n-12 m^{4}+12 m^{3} n-14 m^{3}-$ $4 m^{2} n+6 m^{2}+12 m-8 n$

(c) $G_{1}\left(P_{n}\left[P_{m}\right] R\right)=20 m^{3} n-28 m^{3}+16 m^{2} n-16 m^{2}-$ $12 m n+16 m-6 n$ (d) $G_{2}\left(P_{n}\left[P_{m}\right] R\right)=32 m^{4} n-56 m^{4}+40 m^{3} n-56 m^{3}-$ $20 m^{2} n+36 m^{2}-36 m n+32 m-4 n-4$

For $k=2$,

(a) $G_{1}\left(P_{n} \quad\left[P_{m}\right] S\right)=8 m^{3} n-10 m^{3}+12 m^{2} n-12 m^{2}+$ $4 m n+8 m-6 n+(8 m n-8 m) \sum_{i=1}^{m-1}(m-i)$

(b) $G_{2}\left(P_{n}\left[P_{m}\right] S\right)=8 m^{4} n-12 m^{4}+12 m^{3} n-14 m^{3}+$ $2 m^{2}-8 m n+12 m-8 n+\left(8 m^{2} n-8 m^{2}\right) \sum_{i=1}^{m-1}(m-i)$

(c) $G_{1}\left(P_{n}\left[P_{m}\right] R\right)=20 m^{3} n-28 m^{3}+20 m^{2} n-20 m^{2}-$ $12 m n+16 m-6 n+(8 m n-8 m) \sum_{i=1}^{m-1}(m-i)$ 


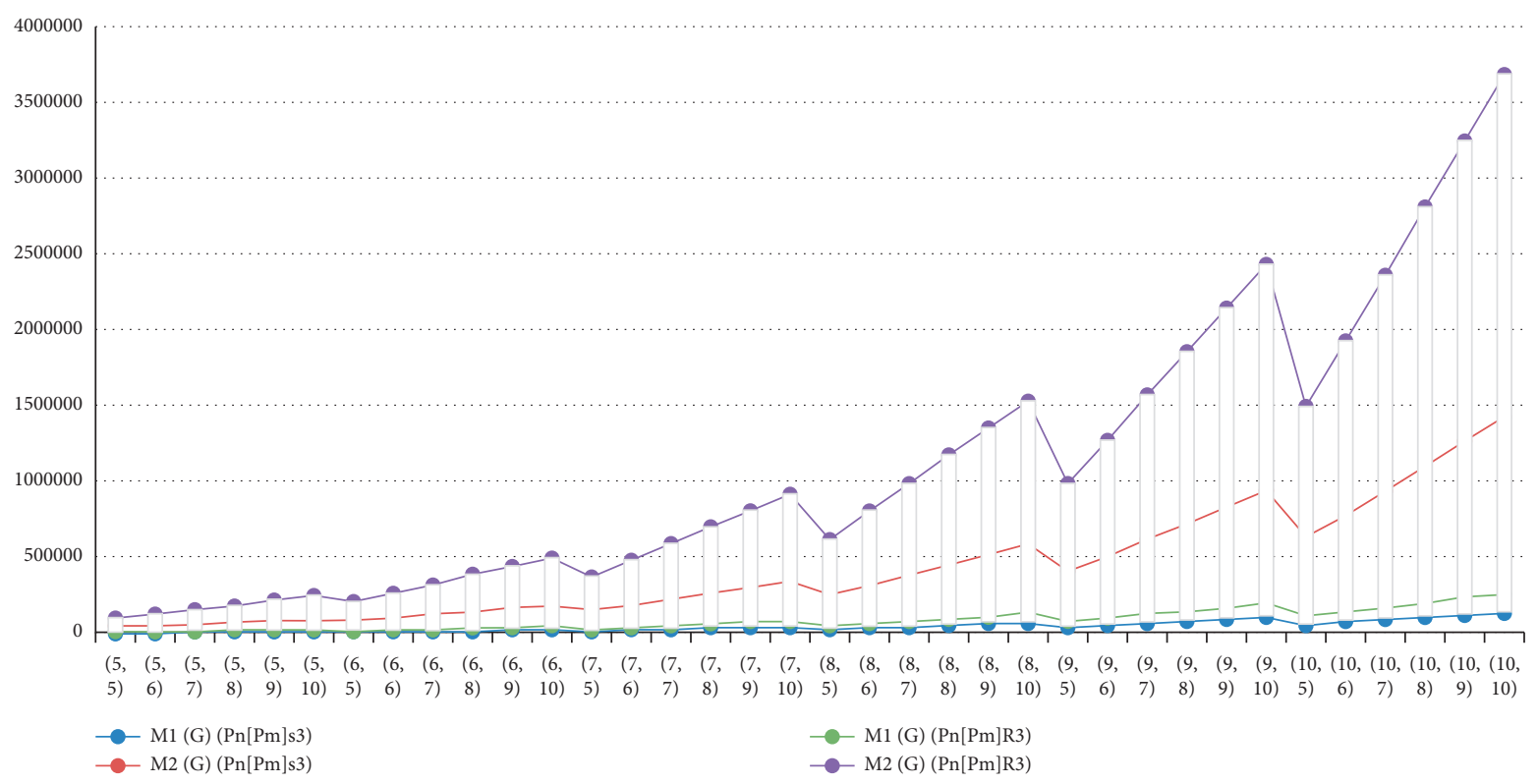

FIGURE 7: Numerical comparison of Zagreb indices of generalized subdivision and semitotal point operations with lexicographic product of the networks for $k=3$.

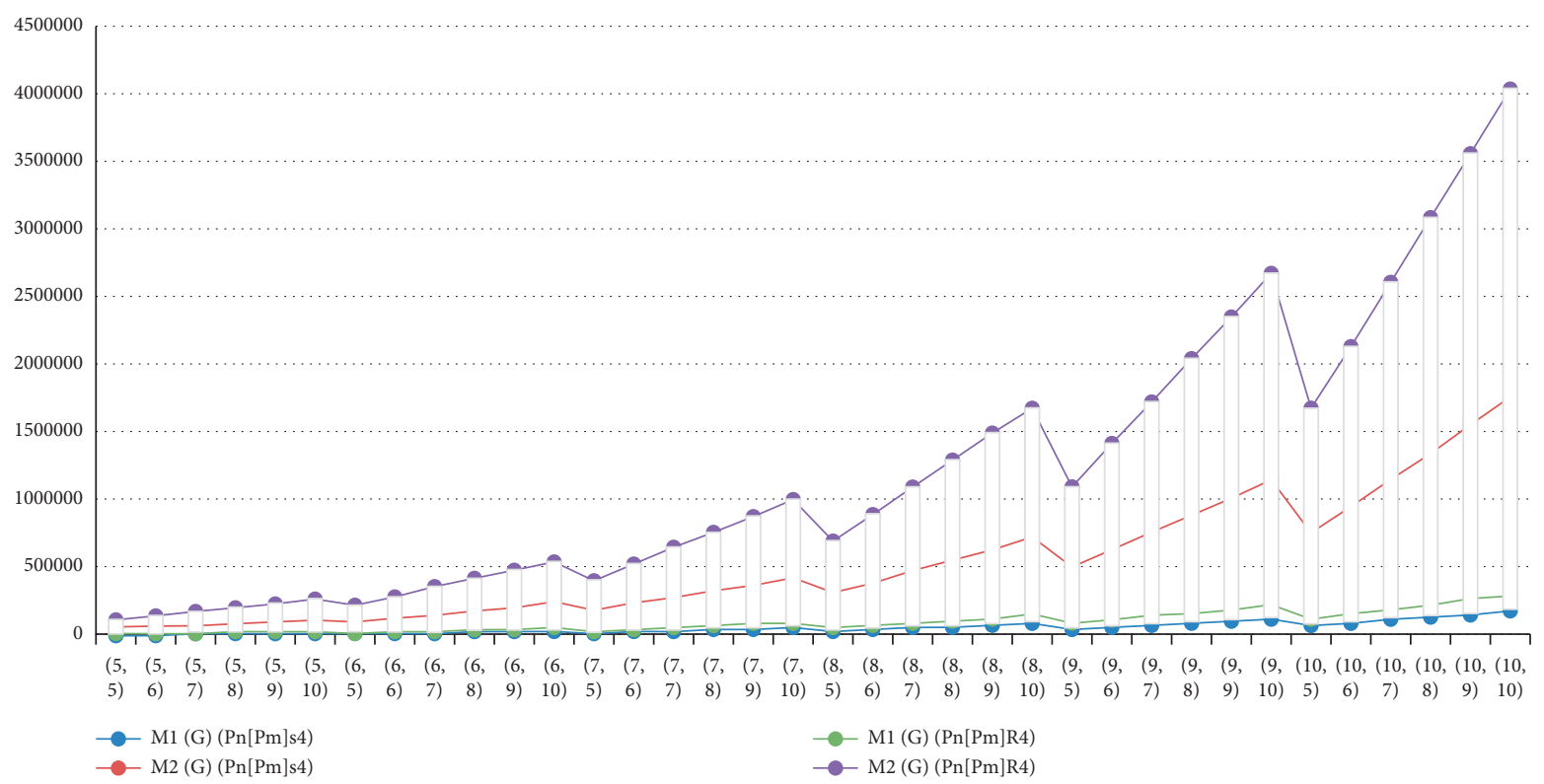

Figure 8: Numerical comparison of Zagreb indices of generalized subdivision and semitotal point operations with lexicographic product of the networks for $k=4$.

(d) $G_{2}\left(P_{n}\left[P_{m}\right] R\right)=32 m^{4} n-56 m^{4}+44 m^{3} n-60 m^{3}-$ $20 m^{2} n+36 m^{2}-36 m n+32 m-4 n-4+\left(8 m^{2} n-\right.$ $\left.8 m^{2}\right) \sum_{i=1}^{m-1}(m-i)$

For $k=3$,

(a) $G_{1} \quad\left(P_{n}\left[P_{m}\right] S\right)=8 m^{3} n-10 m^{3}+16 m^{2} n-16 m^{2}-$ $4 m n+8 m-6 n+(16 m n-16 m) \sum_{i=1}^{m-1}(m-i)$

(b) $G_{2}\left(P_{n} \quad\left[P_{m}\right] S\right)=8 m^{4} n-12 m^{4}+12 m^{3} n-14 m^{3}+$ $4 m^{2} n-2 m^{2}-8 m n+12 m-8 n+\left(16 m^{2} n-16 m\right)$ $\sum_{i=1}^{m-1}(m-i)$ (c) $G_{1}\left(P_{n}\left[P_{m}\right] R\right)=20 m^{3} n-28 m^{3}+28 m^{2} n-28 m^{2}-$ $12 m n+16 m-6 n+(16 m n-16 m) \sum_{i=1}^{m-1}(m-i)$

(d) $G_{2}\left(P_{n}\left[P_{m}\right] R\right)=32 m^{4} n-56 m^{4}+48 m^{3} n-64 m^{3}-$ $20 m^{2} n+36 m^{2}-36 m n+32 m-4 n-4+\left(16 m^{2} n-\right.$ $16 m) \sum_{i=1}^{m-1}(m-i)$

For $k=4$,

(a) $G_{1}\left(P_{n}\left[P_{m}\right] S\right)=8 m^{3} n-10 m^{3}+20 m^{2} n-20 m^{2}-$ $4 m n+8 m-6 n+(24 m n-24 m) \sum_{i=1}^{m-1}(m-i)$ 


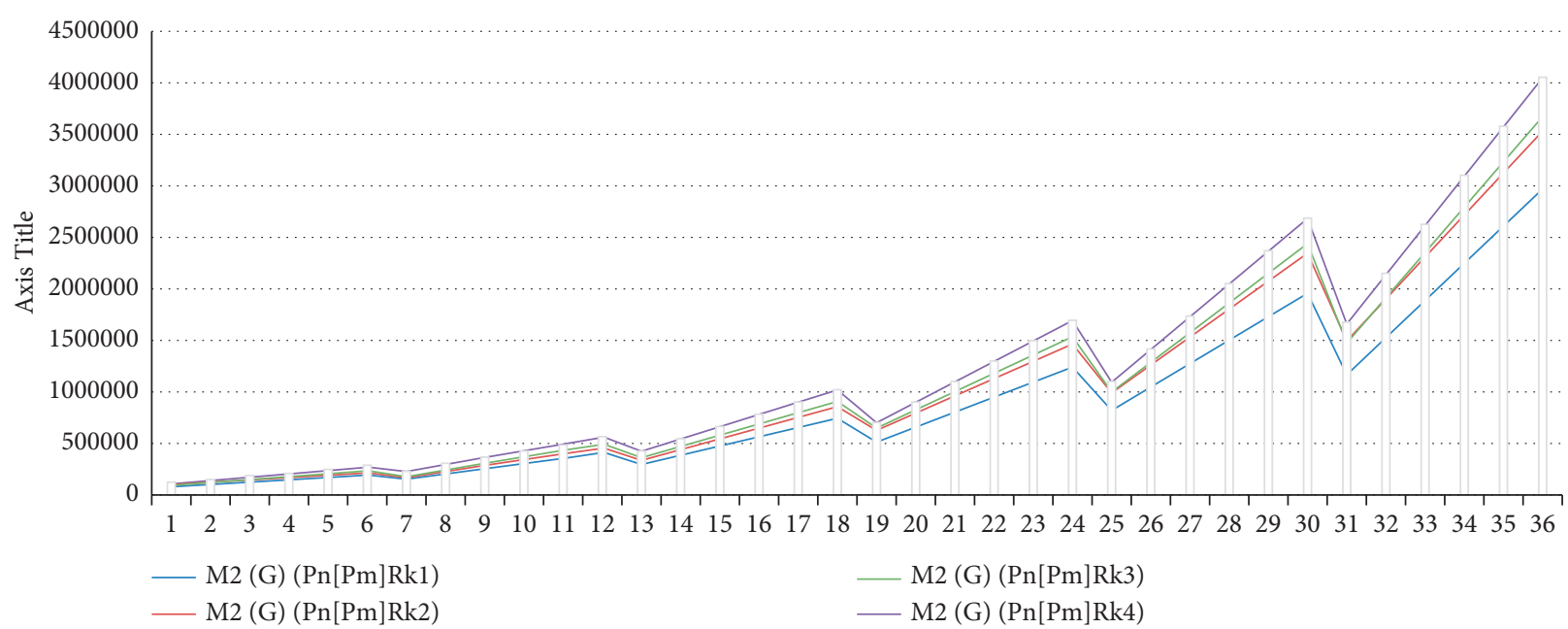

FIGURE 9: Numerical comparison of Zagreb indices of generalized subdivision and semitotal point operations with lexicographic product of the networks for $M_{2}\left(P_{n}\left[P_{m}\right]_{R_{k}}\right)$, where $k \in\{1,2,3,4\}$.

(b) $G_{2}\left(P_{n} \quad\left[P_{m}\right] S\right)=8 m^{4} n-12 m^{4}+12 m^{3} n-14 m^{3}+$ $8 m^{2} n-6 m^{2}-8 m n+12 m-8 n+\left(24 m^{2} n-24 m^{2}\right)$ $\operatorname{sum}(m-i)$

(c) $G_{1}\left(P_{n}\left[P_{m}\right] R\right)=20 m^{3} n-28 m^{3}+32 m^{2} n-32 m^{2}-$ $12 m n+16 m-6 n+(24 m n-24 m) \operatorname{sum}(m-i)$

(d) $G_{2}\left(P_{n}\left[P_{m}\right] R\right)=32 m^{4} n-56 m^{4}+52 m^{3} n-68 m^{3}-$ $20 m^{2} n+36 m^{2}-36 m n+32 m-4 n-4+\left(24 m^{2} n-\right.$ $\left.24 m^{2}\right) \operatorname{sum}(m-i)$

In this paper, we proved the result of 1 st and 2 nd Zagreb indices of networks $G_{1}\left[G_{2}\right]_{T_{k}}$, by using generalized subdivision and generalized semitotal point operation and lexicographic product. The first and second Zagreb indices are in the form $M_{i}\left(G_{1}\left[G_{2}\right]_{T_{k}}\right)$, where $(i=1,2)$. We plot the network of these derived exact values individually and after that plot a combine network for $k \in\{1,2,3,4\}$ to compare these results. In this result, it is clearly shown that the 2 nd Zagreb indices $M_{2}\left(G_{1}\left[G_{2}\right]_{T_{R}}\right)$ shows better result as compared to others' exact values.

\section{Data Availability}

All the data are included within this paper. However, more details of the data can be obtained from the corresponding author upon request.

\section{Conflicts of Interest}

The authors have no conflicts of interest.

\section{Acknowledgments}

This work was supported by the Humanities and School science project of Anhui provincial Education Department: research on social crisis management in Anhui cities under the background of the data (subject no: SK2016A0233).

\section{References}

[1] H. Wiener, "Structural determination of paraffin boiling points," Journal of the American Chemical Society, vol. 69, no. 1, pp. 17-20, 1947.

[2] I. Gutman and N. Trinajstić, "Graph theory and molecular orbitals. Total $\varphi$-electron energy of alternant hydrocarbons," Chemical Physics Letters, vol. 17, no. 4, pp. 535-538, 1972.

[3] D. M. Cvetkocic, M. Doob, and H. Sachs, Spectra of Graphs: Theory and Applications, Academic, New York, NY, USA, 1980.

[4] M. Randic, "Characterization of molecular branching," Journal of the American Chemical Society, vol. 97, no. 23, pp. 6609-6615, 1975.

[5] I. Gutman, "Degree-based topological indices," Croatica Chemica Acta, vol. 86, no. 4, pp. 351-361, 2013.

[6] W. Yan, B.-Y. Yang, and Y.-N. Yeh, "The behavior of Wiener indices and polynomials of graphs under five graph decorations," Applied Mathematics Letters, vol. 20, no. 3, pp. 290-295, 2007.

[7] M. Eliasi and B. Taeri, "Four new sums of graphs and their Wiener indices," Discrete Applied Mathematics, vol. 157, no. 4, pp. 794-803, 2009.

[8] H. Deng, D. Sarala, S. K. Ayyaswamy, and S. Balachandran, "The Zagreb indices of four operations on graphs," Applied Mathematics and Computation, vol. 275, pp. 422-431, 2016.

[9] S. Akhter and M. Imran, "Computing the forgotten topological index of four operations on graphs," AKCE International Journal of Graphs and Combinatorics, vol. 14, no. 1, pp. 70-79, 2017.

[10] Z. S. Mufti, M. F. Nadeem, W. Gao, and Z. Ahmad, “Topological study of the para-line graphs of certain pentacene via topological indices," Open Chemistry, vol. 1, no. 16, pp. 1200-1206, 2018.

[11] A. Adnan, M. F. Nadeem, Z. Zahid, S. Zafar, and W. Gao, "Computing certain topological indices of the line graphs of subdivision graphs of some rooted product graphs," Mathematics, vol. 5, no. 7, pp. 393-401, 2019.

[12] J.-B. Liu, M. Javaid, and H. M. Awais, "Computing zagreb indices of the subdivision-related generalized operations of graphs," IEEE Access, vol. 7, pp. 105479-105488, 2019. 
[13] H. Deng, D. Sarala, S. K. Ayyaswamy, and S. Balachandran, "The Zagreb indices of graphs based on four new operations related to lexicographic product," Applied Mathematics and Computation, vol. 309, pp. 156-169, 2017.

[14] H. M. Awais, M. Javaid, and M. Jamal, "Forgotten Index of generalized F-sum graphs," Journal of Prime Research in Mathematics, vol. 15, pp. 115-128, 2019. 